\title{
Liouville master equation for multielectron dynamics: Neutralization of highly charged ions near a LiF surface
}

\author{
Ludger Wirtz, ${ }^{1,2}$ Carlos O. Reinhold, ${ }^{2,3}$ Christoph Lemell, ${ }^{1}$ and Joachim Burgdörfer ${ }^{1,2,3}$ \\ ${ }^{1}$ Institute for Theoretical Physics, Vienna University of Technology, Wiedner Hauptstrasse 8-10/136, A-1040 Vienna, Austria \\ ${ }^{2}$ Department of Physics and Astronomy, University of Tennessee, Knoxville, Tennessee 37996-1200 \\ ${ }^{3}$ Physics Division, Oak Ridge National Laboratory, Oak Ridge, Tennessee 37831-6372
}

(Received 22 August 2002; published 28 January 2003)

\begin{abstract}
We present a simulation of the neutralization of highly charged ions in front of a lithium fluoride surface including the close-collision regime above the surface. The present approach employs a Monte Carlo solution of the Liouville master equation for the joint probability density of the ionic motion and the electronic population of the projectile and the target surface. It includes single as well as double particle-hole (de)excitation processes and incorporates electron correlation effects through the conditional dynamics of population strings. The input in terms of elementary one- and two-electron transfer rates is determined from classical trajectory Monte Carlo calculations as well as quantum-mechanical Auger calculations. For slow projectiles and normal incidence, the ionic motion depends sensitively on the interplay between image acceleration towards the surface and repulsion by an ensemble of positive hole charges in the surface ("trampoline effect"). For $\mathrm{Ne}^{10+}$ we find that image acceleration is dominant and no collective backscattering high above the surface takes place. For grazing incidence, our simulation delineates the pathways to complete neutralization. In accordance with recent experimental observations, most ions are reflected as neutral or even as singly charged negative particles, irrespective of the charge state of the incoming ions.
\end{abstract}

DOI: 10.1103/PhysRevA.67.012903

PACS number(s): 34.50.Dy, 34.70.+e, 79.20.Rf

\section{INTRODUCTION}

The fabrication of clean surfaces under ultrahigh vacuum conditions has stimulated experimental studies of the interaction of ions with surfaces. Possible technological applications include surface diagnostics and surface modification, e.g., edging of microstructures. Apart from interpreting experimental results, the theory of ion-surface interaction poses an interesting challenge because of its intrinsic many-body character and its interdisciplinary connections to different subfields of physics. Methods from solid-state physics, molecular physics (quantum chemistry), and atomic collision physics are applied in order to describe the charge exchange between surface and projectile and the ejection of secondary particles $[1,2]$. Only a few simple model systems are currently amenable to full quantum-mechanical ab initio calculations (see, e.g., Refs. [3-5]). For more complicated systems, the challenge to theory consists in finding realistic simplifications and approximations [6-9]. In the case of a highly charged ion approaching a metal surface, a classical description of charge transfer within the framework of the "classical over the barrier" model has turned out to be quite successful [6].

The interaction of ions with insulator surfaces adds additional degrees of complexity. Electron transfer from an insulator surface to the projectile ion can lead to a local microscopic charge up of the surface. Through self-trapping of electronic defects, the recombination energy of the projectile ion (i.e., the potential energy that the ion carries into the collision with the surface) can be converted into kinetic energy of surface atoms and can lead to the ablation of secondary particles from the surface (potential sputtering [10]). Fur- thermore, the local positive charge at the surface influences the projectile dynamics. In fact, Briand et al. [11] put forward the intriguing hypothesis that the repulsive interaction between the holes and the projectile can become stronger than the attractive self-image force and eventually lead to the backscattering of a still multiply charged ion high above the surface without touching it. This "trampoline effect" (Fig. 1) should be distinguished from the more conventional backscattering due to close binary collisions with surface ions as well as from scattering at the macroscopically charged-up surfaces. Figure 1 also illustrates that electron capture at large distances from the surface leads to the population of Rydberg states of the projectile. Since some of the inner shells are unoccupied, such a transient state is called a hollow atom/ion $[12,13]$. Since conduction electrons in metals are delocalized, the system has approximate cylindrical symmetry, and simple analytical estimates of the rates for electron transfer over the potential barrier between the surface and the projectile can be obtained [6]. For ions in front of an insulator surface such as $\mathrm{LiF}$ [Fig. 1(b)], the modeling of charge-transfer events is far more involved. In this case, valence electrons are localized around ionic centers and the lack of symmetry of the electronic potential impedes a simple determination of electron transfer rates. During the approach of the ion to the surface, hollow atoms decay by electron loss to the surface, intra-atomic Auger processes, and level promotion. This, in turn, opens up the possibility of transfer of valence electrons into more tightly bound states of the projectile. The formation of electron holes in the surface reduces the capture rate of electrons unless the holes diffuse through the crystal. Therefore, both the mobility of holes in the crystal and the subsequent capture of more tightly bound 

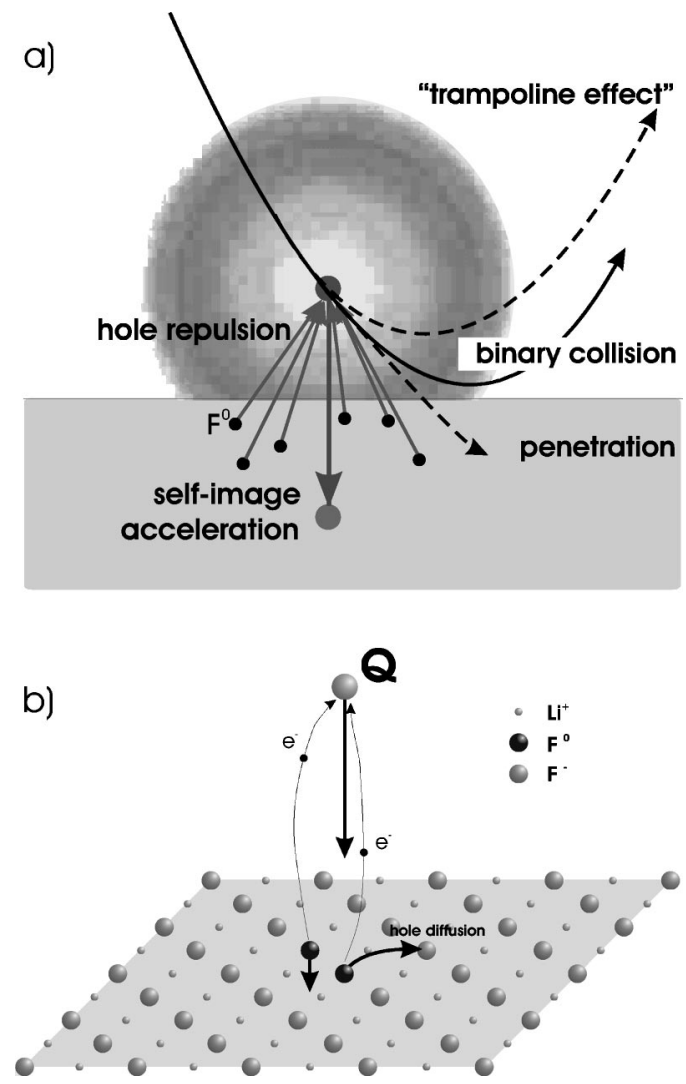

FIG. 1. (a) Schematic diagram illustrating different possible ion trajectories: penetration, reflection via a binary collision with a topmost atom at the surface, and reflection at large distances due to the repulsion by the holes created at the surface. The figure also illustrates the formation of a hollow atom/ion in front of the surface (the cloud around the ion corresponds to the charge density of electrons transferred from the surface to the ion). The ion is attracted to the surface by the interaction with its own image while the holes in the surface cause a repulsive force onto the projectile nucleus that is only partially screened by the electrons in shells with high quantum numbers. (b) Subset of processes occurring during the approach of a multiply charged ion towards a LiF surface: electron capture, electron loss, hole formation, and hole diffusion.

electrons from the same ionic site have to be included in a realistic description for insulator surfaces.

In this paper, we extend previous analyses $[7,14]$ of ion neutralization in front of insulator surfaces to include several additional processes: (i) multiple sequential electron transfer of electrons from and to the same fluorine site, (ii) interatomic Auger transitions, and (iii) the interplay of hole mobility and charge transfer. Our analysis is based on an explicit treatment of the multielectron dynamics within the framework of a Liouville master equation. It allows for an approximate treatment of the correlated dynamics of hole formation in the solid and multiple excitation in the projectile by means of a Monte Carlo event sampling. This description goes beyond the previously employed rate equations for single-particle expectation values as it deals directly with the joint probability density of projectile and target degrees of freedom. This allows us to make predictions for the existence of a trampoline effect (or the lack thereof) for $\mathrm{LiF}$ as well as for the neutralization of multiply charged ions in grazing incidence scattering. In Sec. II we give an overview over the theoretical framework of the Liouville master equation. Sections III-VI are devoted to the calculation of the interaction potentials as well as of rates for various electronic processes controlling the dynamics of ion-surface interactions. Even though an ab initio calculation of the rates appears not feasible at this moment, we attempt to provide estimates of all rates entering our simulation that are free of adjustable parameters. We also disentangle the processes that were previously called "side feeding." This term was originally introduced as an additional mechanism for electron transfer into inner shells in order to explain the rapid neutralization and relaxation seen in the experiments $[15,16]$. We identify side feeding for $\mathrm{LiF}$ as originating both from resonant sequential electron capture from the same F site and from Auger processes. As first applications, we will present in Sec. VII simulations for both vertical and grazing incidence of a highly charged $\mathrm{Ne}^{10+}$ ion. Atomic units are used throughout unless stated otherwise.

\section{THEORY}

\section{A. Rate equations}

We begin by briefly reviewing previously employed rate equation methods for mean values of observables such as the mean occupation $\mathcal{P}_{n}=\left\langle P_{n}\right\rangle$ of the $n$th shell of the incident projectile. For an extensive analysis of the different processes that enter the ion-surface interaction, we refer the reader to earlier reviews (e.g., Refs. [1,2]). Here, we briefly recall the generally accepted scenario. At large distances, electrons are transferred from the valence band of the surface to the projectile by resonant capture $(C)$ into outer shells of the projectile, leading to a highly unstable hollow ion/atom. This system decays by resonant electron loss $(L)$ to the surface and by intra-atomic Auger decay [autoionization (AI)]. For light projectiles, radiative decay rates are several orders of magnitude smaller than typical Auger rates, and can be safely neglected. Close to the surface, additional interatomic Auger processes may take place, namely, Auger capture (AC) and Auger deexcitation (AD). During AC one electron from the surface is transferred to an inner shell of the projectile and the excess energy transfers another electron from the surface to the continuum. AD denotes the demotion of an electron from a higher projectile state to a lower projectile state with the simultaneous emission of an electron from the surface. Finally, for very close distances to the surface, resonant capture from core electrons may play an important role. As will be described below, for LiF surfaces the latter can be ignored.

The interaction of a highly charged ion with a surface is a true multielectron problem. Drastic approximations are therefore inevitable to reach a starting point for quantitative simulations. The first step consists of breaking down the multielectron problem into a sequence of one-electron and two-electron processes. Resonant electron capture and loss are one-electron processes (or single electron-hole pair excitations), whereas Auger transitions correspond to twoelectron processes (or double electron-hole pair excitations). 
Additionally, effects of collective screening by "passive" electrons need to be included. A second step is the use of the fixed ion approximation (FIA) [17], within which rates for all electron processes are calculated in the limit of zero velocity $(\dot{\vec{R}}=0)$ of the projectile, i.e., they are assumed to be only parametrically dependent on the position of the ion, $\vec{R}$. The latter is justified only for slow collisions. As will be discussed below, grazing incidence scattering with slow perpendicular but fast parallel motion falls somewhat outside of the scope of the FIA. For surfaces with approximate translational symmetry in the surface plane such as metals, this latter case can be treated within the framework of a Galilei shift of the conduction band [2,18-20]. No such simplification is available for ionic crystals. We therefore restrict ourselves to the simulation of collision systems with velocities $v \leqq 0.1$ a.u. for which the FIA is approximately valid.

Rate equations for ion-metal surfaces describe the evolution in terms of mean values $\mathcal{P}_{n}=\left\langle P_{n}\right\rangle$ of the set $\left\{P_{n}\right\}$ of populations $P_{n}$ of the $n$ shells, where $n$ is the principal quantum number and $0<P_{n}<2 n^{2}$. The evolution of $\mathcal{P}_{n}$ is coupled to that of the average position vector $\vec{R}$ $=\left(R_{x}, R_{y}, R_{z}\right)$ and velocity vector $\dot{\vec{R}}$, of the ion (we use a coordinate system whose origin is at a surface $\mathrm{F}$ atom such that the $x$ and $y$ axes are parallel to the surface and the $z$ axis is perpendicular to the surface). The dynamics of $\vec{R}, \dot{\vec{R}}$, and $\left\{\mathcal{P}_{n}\right\}$ is governed by a system of coupled rate equations involving the transition rates for the various processes, $\gamma^{C}, \gamma^{L}$, $\gamma^{A I}, \gamma^{A C}$, and $\gamma^{A D}$,

$$
\begin{aligned}
\frac{d}{d t} \mathcal{P}_{n}= & \gamma_{n}^{C}(\vec{R})+\gamma_{n}^{A C}(\vec{R})-\gamma_{n}^{L}(\vec{R}) \mathcal{P}_{n}+\sum_{n^{\prime}>n}\left(\gamma_{n^{\prime}, n}^{A D}(\vec{R}) \mathcal{P}_{n^{\prime}}\right. \\
& \left.+\frac{1}{2} \gamma_{n^{\prime}, n}^{A I} \mathcal{P}_{n^{\prime}}^{2}\right)-\sum_{n^{\prime}<n}\left[\gamma_{n, n^{\prime}}^{A D}(\vec{R}) \mathcal{P}_{n}+\gamma_{n, n^{\prime}}^{A I} \mathcal{P}_{n}^{2}\right],
\end{aligned}
$$

coupled to Hamilton's equation for the average ion trajectory,

$$
\begin{gathered}
\frac{d}{d t} \vec{R}=\dot{\vec{R}}, \\
\frac{d}{d t} \dot{\vec{R}}=-\frac{1}{M} \nabla_{\vec{R}} V_{p}\left(\vec{R},\left\{\mathcal{P}_{n}\right\}\right),
\end{gathered}
$$

where $M$ is the mass of the ion and $V_{p}$ is the effective interaction potential of the ion with the surface, which depends on the set of average populations, $\left\{\mathcal{P}_{n}\right\}$. Details of the rates appearing in Eq. (2.1) will be discussed below. One of the fundamental assumptions underlying Eqs. (2.1)-(2.3) is that the state of the surface remains unchanged during the scattering process and remains decoupled from the dynamics of the internal state of the projectile. This is based on the assumptions that (i) holes are refilled quasi-instantaneously and (ii) excess charges that enter the conduction band through electron-loss processes are carried away equally fast. These assumptions can be justified for metals with typical short relaxation times of the order of the inverse plasmon frequency $\tau_{r} \approx \omega_{p}^{-1}$. Clearly, for insulator surfaces such as alkali halides $(\mathrm{LiF})$ discussed in the following, this approximation breaks down and the coupling of the projectile and target electronic degrees of freedom should be taken into account. The rate equation approach for mean values, i.e., for onepoint functions, is clearly inadequate; and instead, a formulation containing the information on many-point correlations is desired.

\section{B. Liouville master equation}

We formulate now the problem of conditional dynamics in this multielectron system in terms of the joint phase-space probability density $\rho\left(t, \vec{R}, \dot{\vec{R}},\left\{P^{(P)}\right\},\left\{P^{(F)}\right\}\right)$. It depends on the phase-space coordinates $(\vec{R}, \dot{\vec{R}})$ of the projectile as well as on the "string" of integer occupation numbers characterizing the internal state of the projectile,

$$
\left\{P^{(P)}\right\}=\left\{P_{1}^{(P)}, \ldots, P_{n_{\max }}^{(P)}, P_{I}\right\},
$$

where $n_{\max }$ is the maximum $n$ shell considered,

$$
0 \leqslant P_{n}^{(P)} \leqslant 2 n^{2}
$$

and $P_{I}$ denotes the occupation number of continuum states (ionized electrons). Furthermore, it depends on the strings of occupation numbers of localized hole states in the $2 p$ orbitals of the various surface fluorines:

$$
\left\{P^{(F)}\right\}=\left\{P_{0,0,0}^{(F)}, P_{1,0,0}^{(F)}, \ldots, P_{\vec{a}}^{(F)}\right\} .
$$

Deeper lying shells, e.g., F (2s), are not expected to provide important contributions because of their increased binding energy that suppresses over-barrier transitions and, in addition, because of their small statistical weight compared to that of the $2 p$ subshell. The subscript denotes the lattice site $\vec{a}=(i, j, k)$ of the hole in the sublattice of fluorine sites with

$$
0 \leqslant P_{a}^{(F)} \leqslant 6 .
$$

The above description in terms of localized holes holds, because the valence band of $\mathrm{LiF}$ is, to a good approximation, represented by occupied $2 p$ orbitals of F. Inner-shell contributions can be safely neglected. We, furthermore, neglect the conduction band that lies above the vacuum ionization threshold as well as the presence of localized surface excitonic states. That is, the loss channel included represents an electron lost from the projectile, which recombines with a hole in the valence band. In line with most previous rate equation models $[6,8]$, we only distinguish populations of different principal shells of the projectile, $P_{n}$, in Eq. (2.4) without differentiating among $(n l)$ subshell populations or individual configurations $\left(n l, n^{\prime} l^{\prime}, \ldots\right)^{2 s+1} L$ (for an exception, see Ref. [21]). Clearly, this is a drastic simplification that can only be justified in that the extracted observables contain averages over subshells and relevant transition rates to be discussed below are relatively insensitive to the individual configurations. Because of the dependence of $\rho$ on the 
phase-space coordinates $(\vec{R}, \dot{\vec{R}})$ of the projectile as well as the state space variables $P_{n}^{(P)}, P_{a}^{(F)}$ rather than only the mean values, it contains the information on the conditional dynamics of the projectile population in the presence of target excitations. In principle, the multielectron dynamics can be described by a hierarchy of $N$-point correlation functions, an example of which are two-point functions $\left\langle P_{n} P_{n^{\prime}}\right\rangle$. The information on the complete set of $N$-point correlation functions is included in $\rho$. The equation of motion of $\rho$ is of the form of a Liouville master equation

$$
\left[\frac{\partial}{\partial t}+\dot{\vec{R}} \cdot \vec{\nabla}_{\vec{R}}-\frac{1}{M}\left(\vec{\nabla}_{\vec{R}} V_{p}\right) \cdot \vec{\nabla}_{\vec{R}}\right] \rho=\operatorname{R} \rho
$$

where the "relaxation" (collision) operator $\mathbb{R}$ is given by

$$
\begin{aligned}
\operatorname{R} \rho= & \sum_{\left\{P^{\prime}(P)\right.} \sum_{\left\{P^{\prime}(F)\right\}}\left[\Gamma\left(\vec{R}_{j},\left\{\Delta P^{(P)}\right\},\left\{\Delta P^{(F)}\right\}\right) \rho^{\prime}\right. \\
& \left.-\Gamma\left(\vec{R}_{j},\left\{-\Delta P^{(P)}\right\},\left\{-\Delta P^{(F)}\right\}\right) \rho\right] .
\end{aligned}
$$

In Eq. (2.9), the following shorthand notation for the transition rates between joint projectile and target strings have been used:

$$
\begin{aligned}
\Gamma\left(\vec{R},\left\{\Delta P^{(P)}\right\},\left\{\Delta P^{(F)}\right\}\right) \\
\quad=\Gamma\left(\vec{R},\left\{P^{\prime(P)} \rightarrow P^{(P)}\right\},\left\{P^{\prime(F)} \rightarrow P^{(F)}\right\}\right),
\end{aligned}
$$

$$
\begin{aligned}
\Gamma\left(\vec{R},\left\{-\Delta P^{(P)}\right\},\left\{-\Delta P^{(F)}\right\}\right) \\
\quad=\Gamma\left(\vec{R},\left\{P^{(P)} \rightarrow P^{\prime(P)}\right\},\left\{P^{(F)} \rightarrow P^{\prime(F)}\right\}\right) .
\end{aligned}
$$

The transition rates are dependent on the local position of the ion, $\vec{R}$, but are assumed to be independent of $\overrightarrow{\vec{R}}$ in line with the fixed-ion approximation. $\rho^{\prime}(\rho)$ in Eq. (2.9) denotes the density at the (un)primed "coordinates" of the string. The effective projectile potential $V_{p}$ that governs the ionic motion will depend, in general, on the strings as well, i.e., $V_{p}$ $=V_{p}\left(\vec{R},\left\{P^{(P)}\right\},\left\{P^{(F)}\right\}\right)$. In the transition rates $\Gamma$ of Eq. (2.10), we include single $\left(\Gamma^{(1)}\right)$ and double $\left(\Gamma^{(2)}\right)$ particlehole (de)excitation processes,

$$
\begin{aligned}
\Gamma\left(\vec{R},\left\{\Delta P^{(P)}\right\},\left\{\Delta P^{(F)}\right\}\right)= & \Gamma^{(1)}\left(\vec{R},\left\{\Delta P^{(P)}\right\},\left\{\Delta P^{(F)}\right\}\right) \\
& +\Gamma^{(2)}\left(\vec{R},\left\{\Delta P^{(P)}\right\},\left\{\Delta P^{(F)}\right\}\right),
\end{aligned}
$$

where $\Gamma^{(1)}$ contains the contribution from resonant capture $C$, resonant loss $L$, hole hopping $H$, and ionization by promotion through the continuum $I$, while $\Gamma^{(2)}$ includes the contribution from $\mathrm{AC}, \mathrm{AD}$, and $\mathrm{AI}$, i.e.,

$$
\Gamma^{(1)}=\Gamma^{C}+\Gamma^{L}+\Gamma^{H}+\Gamma^{I},
$$

$$
\Gamma^{(2)}=\Gamma^{A C}+\Gamma^{A D}+\Gamma^{A I} .
$$

We have dropped the arguments for the dependence on the phase space coordinates as well as on the strings for brevity. Consider, for example, capture $C$. In this case, the change of the strings in the transition rate $\Gamma^{C}\left(\vec{R},\left\{\Delta P^{(P)}\right\},\left\{\Delta P^{(F)}\right\}\right.$ are constrained by

$$
\begin{aligned}
& \sum_{n=1}^{n_{\max }} \Delta P_{n}^{(P)}=\sum_{n=1}^{n_{\max }}\left(P_{n}^{(P)}-P_{n}^{\prime(P)}\right)=+1, \\
& \sum_{\vec{a}} \Delta P_{\vec{a}}^{(F)}=\sum_{\vec{a}}\left(P_{\vec{a}}^{(F)}-P_{\vec{a}}^{\prime(F)}\right)=-1 .
\end{aligned}
$$

The sum in Eq. (2.14a) extends only up to $n \leqslant n_{\max }$, but excludes the $I$ component. Constraints analogous to Eq. (2.14) can be given for all other processes indicated by Eqs. (2.12) and (2.13) (see below). Clearly, determination of this multitude of rates requires a wide array of additional and, in part, drastic approximations discussed in the following sections.

\section{Monte Carlo solution}

Direct integration of the Liouville master equation [Eq. (2.8)] appears to be extremely difficult in view of the large number of degrees of freedom involved. We employ, instead, a Monte Carlo sampling technique for ensembles of stochastic realizations of trajectories. We follow a large number of ionic trajectories with identical initial conditions for the phase-space variables $R, \dot{R}$ as well as for the strings along an event-by-event sequence of stochastic electronic processes whose probability laws are governed by the rates of the underlying Liouville master equation.

In practice, the time integration is carried out by propagating the system during small time steps $\Delta t$ and taking the limit $\Delta t \rightarrow 0$ such that at most a single electronic transition can take place during this time period. The probability for any process with transition rate $\Gamma^{\alpha}$ to occur within a time interval $\Delta t$ is determined by

$$
W^{\alpha}(\Delta t)=1-\exp \left(-\Delta t \Gamma^{\alpha}\right) .
$$

In order to decide which electronic transition occurs (if any) during the time period $\Delta t$, we use the so-called rejection method for the distribution equation (2.15). A random number $r$ is generated uniformly in the interval $(0,1)$ for each transition. If $r<W^{\alpha}(\Delta t)$, the process is assumed to take place and $P_{n}^{(P)}$ and $P^{(F)}$ are then adjusted,

$$
\begin{aligned}
& \Delta P_{n}^{(P)}=P_{n}^{(P)}(t+\Delta t)-P_{n}^{(P)}(t)= \pm 0,1,2, \\
& \Delta P_{a}^{(F)}=P_{a}^{(F)}(t+\Delta t)-P_{a}^{(F)}(t)= \pm 0,1,2,
\end{aligned}
$$

depending on the process $\alpha$ under consideration. At the same time, the coordinate and velocity of the HCI are propagated in time according to

$$
\vec{R}(t+\Delta t)=\dot{\vec{R}}(t) \Delta t
$$




$$
\dot{\vec{R}}(t+\Delta t)=-\frac{1}{M} \vec{\nabla}_{\vec{R}} V_{p}\left[\vec{R}(t),\left\{P_{n}^{(P)}(t)\right\},\left\{P_{a}^{(F)}(t)\right\}\right] \Delta t .
$$

The resulting functions $\left\{P_{n}^{(P)}(t)\right\}_{\mu}$ and $\left\{P_{\vec{a}}^{(F)}(t)\right\}_{\mu}$ for a single stochastic trajectory $\mu$ are discontinuous functions of time. After sampling of a large number of trajectories, $N_{i o n}$, one obtains ensemble averages, e.g.,

$$
\begin{gathered}
\mathcal{P}_{a}^{(F)}(t)=\frac{1}{N_{\text {ion }}} \sum_{\mu=1}^{N_{\text {ion }}}\left[P_{a}^{(F)}(t)\right]_{\mu}, \\
\mathcal{P}_{n}^{(P)}(t)=\frac{1}{N_{\text {ion }}} \sum_{\mu=1}^{N_{\text {ion }}}\left[P_{n}^{(P)}(t)\right]_{\mu} \\
\vec{R}(t)=\frac{1}{N_{\text {ion }}} \sum_{\mu=1}^{N_{\text {ion }}}[\vec{R}(t)]_{\mu}, \quad \dot{\vec{R}}(t)=\frac{1}{N_{\text {ion }}} \sum_{\mu=1}^{N_{\text {ion }}}[\dot{\vec{R}}(t)]_{\mu} .
\end{gathered}
$$

Note that, in general, an additional average over different initial lateral coordinates $\vec{R}_{\|}$of the initial vector $\vec{R}$ $=\left(R_{z}, \vec{R}_{\|}\right)$over the surface unit cell of $\mathrm{LiF}$ is required. Clearly, the solution of the rate equations [Eq. (2.1)] represents a special case of Eqs. (2.19) and (2.20) when (i) the rates entering Eq. (2.8) are physically equivalent to those entering Eq. (2.1) and, more importantly, (ii) the conditional dynamics, i.e., correlations between the population dynamics of the projectile, the target populations ("strings"), and the ionic motion can be neglected. In the following sections, we discuss the approximations and assumptions underlying our choice for the input of the Liouville master equation.

\section{EFFECTIVE POTENTIALS}

The ionic trajectory as well as the charge-transfer dynamics in the projectile ion and in the target surface are determined by effective potentials that incorporate collective screening and polarization effects. Their description requires a number of simplifying assumptions to be discussed in the following sections.

\section{A. Projectile potential}

We consider first the effective interaction potential $V_{p}$, referred to in the following as the projectile potential, which governs the motion of the impinging ion. It can be written as

$$
\begin{aligned}
V_{p}\left(\vec{R},\left\{P^{(P)}\right\},\left\{P^{(F)}\right\}\right)= & V_{p}^{g}\left(\vec{R},\left\{P^{(P)}\right\}\right)+V_{p}^{H}\left(\vec{R},\left\{P^{(P)}\right\},\left\{P^{(F)}\right\}\right) \\
& +V_{p}^{S I}\left(R_{z},\left\{P^{(P)}\right\}\right) .
\end{aligned}
$$

The three contributions in Eq. (3.1) refer to the interaction with the surface in its ground state, $V_{p}^{g}$, to the interaction with the hole excitations in the surface, $V_{p}^{H}$, and the selfinduced polarization (or self-image) of the surface, $V^{S I}$. These interaction potentials depend not only on the local coordinate of the ion, but also on the internal state of the ion and the crystal, i.e., the strings.
The potential $V_{p}^{g}$ contains the interactions of the ion with all sites of the LiF crystal with a lattice constant $d$ $=3.8$ a.u. Two contributions to $V_{p}^{g}$ need to be considered, the long-range potential $V^{\text {ionic }}$ of the ionic cores with the charges $\pm q_{0}$ of the $\mathrm{Li}^{+}$and $\mathrm{F}^{-}$ionic centers (we use the value $q_{0}=0.86$ of Wang et al. [22]) and the short-ranged atomic potential $V^{\text {atomic }}$,

$$
V_{p}^{g}(\vec{R})=V_{p}^{i o n i c}(\vec{R})+V_{p}^{\text {atomic }}(\vec{R})
$$

with

$$
V_{p}^{\text {ionic }}(\vec{R})=\sum_{\vec{a}} \frac{Z q_{\vec{a}}}{|\vec{R}-d \vec{a}|}=\sum_{L i} \frac{Z q_{0}}{\left|\vec{R}-\vec{R}_{L i}\right|}-\sum_{F} \frac{Z q_{0}}{\left|\vec{R}-\vec{R}_{F}\right|}
$$

and

$$
V_{p}^{\text {atomic }}(\vec{R})=\sum_{L i} V^{L i}\left(\left|\vec{R}-\vec{R}_{L i}\right|\right)+\sum_{F} V^{F}\left(\left|\vec{R}-\vec{R}_{F}\right|\right),
$$

where we have assumed a fully stripped projectile with nuclear charge $Z$ (for a partially screened projectile $Z$ is replaced by an effective charge $Q_{\text {eff }}$ defined below). In Eq. (3.3), $q_{\vec{a}}= \pm q_{0}$ and $d \vec{a}=\vec{R}_{L i}$ or $d \vec{a}=\vec{R}_{F}$ denote the position vectors of the ionic charges in the crystal, and are located at regular lattice sites $\vec{a}=(i, j, k)$. We will use in the following the same symbol $\vec{a}$ for lattice vectors at the surface $(k=0)$ and in the bulk $(k \neq 0)$. For the atomic potentials $V^{L i}$ and $V^{F}$, we use a Moliere form [23]. It should be noted that the ground-state potential $V_{p}^{g}$ is short ranged despite the presence of ionic potentials [Eq. (3.3)], because of the overall charge neutrality. Long-range potentials result from hole excitation and polarization (virtual collective excitations).

The effective interactions with the holes are given by the sum over all $F$ sites at lattice coordinates $\vec{a}_{h}$ with a hole present,

$$
\begin{aligned}
V_{p}^{H}\left(\vec{R},\left\{P^{(P)}\right\},\left\{P^{(F)}\right\}\right) \\
\quad=(1-\chi) \sum_{\vec{a}_{h}} \frac{P_{\vec{a}_{h}}^{(F)} Q_{e f f}\left(\left|\vec{R}-d \vec{a}_{h}\right|,\left\{P^{(P)}\right\}\right)}{\left|\vec{R}-d \vec{a}_{h}\right|} .
\end{aligned}
$$

The effective distance $(D)$-dependent charge of the projectile, taking into account incomplete screening, is given by

$$
Q_{e f f}\left(D,\left\{P^{(P)}\right\}\right)=Z-\sum_{\left\{n: r_{n}<D\right\}} P_{n}^{(P)},
$$

where the shell radius $r_{n}$ is approximately given by

$$
r_{n}=n^{2} / q_{n} .
$$

For an effective charge for a given shell $n$ of a projectile, $q_{n}$, we use Slater's rules [24]:

$$
q_{n}=Z-\sum_{n^{\prime} \leqslant n} S_{n, n^{\prime}} P_{n^{\prime}}^{(P)}
$$


with

$$
S_{n, n^{\prime}}=\left\{\begin{array}{rll}
1 & : & n^{\prime}<n-1 \\
0.85 & : & n^{\prime}=n-1 \\
0.35 & : & n^{\prime}=n .
\end{array}\right.
$$

The prefactor $(1-\chi)$ in Eq. (3.5) represents the partial screening of the holes due to the polarization of the surrounding crystal, expressed in terms of the dielectric response function $\chi$. For a detailed discussion of $\chi$, we refer the reader to Ref. [7]. In line with the FIA, we use the static value $(\omega=0)$,

$$
\chi(\omega=0)=\frac{\epsilon(0)-1}{\epsilon(0)+1} .
$$

For $\operatorname{LiF}, \epsilon(0) \approx 9.1$ and $\chi(0)=0.8$.

Finally, the highly charged ion polarizes the LiF surface, which leads to an effective interaction of the projectile with its own "image." At large distances from the surface, the potential takes the proper asymptotic form,

$$
V_{p}^{S I}\left(R_{z},\left\{P^{(P)}\right\}\right) \underset{R_{z} \rightarrow \infty}{\rightarrow}-\frac{\chi Q_{e f f}\left(R_{z},\left\{P^{(P)}\right\}\right)}{4 R_{z}} .
$$

Close to the surface, Eq. (3.11) becomes invalid. We choose an interpolation form that leads to a constant value for $R_{z}$ $\rightarrow 0$ (or equivalently, to a vanishing image acceleration) and to Eq. (3.11) at large distances,

$$
\begin{aligned}
V_{p}^{S I}\left(R_{z},\left\{P^{(P)}\right\}\right)= & -\chi Q_{e f f}\left(R_{z},\left\{P^{(P)}\right\}\right) \\
& \times\left[\left(\frac{1}{4 R_{z_{0}}}\right)^{-5}+\left(\frac{1}{4 R_{z}}\right)^{-5}\right]^{-1 / 5} .
\end{aligned}
$$

Equation (3.12) implicitly contains the information on the location of the image plane defined as

$$
R^{i m}=\lim _{R_{z} \rightarrow \infty}\left(-R_{z}\left[1+4 R_{z} V_{p}^{S I}\left(R_{z},\left\{P^{(P)}\right\}\right)\right]\right)=0,
$$

i.e., the image plane coincides with the topmost layer of the crystal. The distance $R_{z_{0}}$ characterizing the transition between the two limiting forms is chosen to be the distance between two neighboring $\mathrm{F}$ sites, $R_{z_{0}}=\sqrt{2} d$. The physical reason for this choice is the fact that at distances smaller than the lattice spacing, the buildup of a polarization charge should saturate. The particular form of the interpolating function does not influence the results.

\section{B. Effective one-electron potentials}

A detailed discussion of effective one-electron potentials entering the calculation of the electronic transition rates for the present system has been given in Ref. [7]. The extension to sequential multiple capture requires a few additional ingredients to be discussed in the following. The total effective one-electron potential determining the dynamics of the electron at a given position of the ion and a given internal state of the ion and of the surface can be written as

$$
\begin{aligned}
V_{e}\left(\vec{r}, \vec{R},\left\{\widetilde{P}^{(F)}\right\},\left\{\widetilde{P}^{(P)}\right\}\right)= & V_{t e}\left(\vec{r},\left\{\widetilde{P}^{(F)}\right\}\right)+V_{p e}\left(\vec{r}, \vec{R},\left\{\widetilde{P}^{(P)}\right\}\right) \\
& +V_{p e}^{I}\left(\vec{r}, \vec{R},\left\{\widetilde{P}^{(P)}\right\}\right)
\end{aligned}
$$

In Eq. (3.14), $\vec{r}$ denotes the position coordinate of the active electron with respect to the active fluorine site (here taken to be at $\vec{a}=\overrightarrow{0}$ ). The interaction potential between the active electron and the target, $V_{t e}$, can be decomposed as

$$
V_{t e}\left(\vec{r},\left\{\widetilde{P}^{(F)}\right\}\right) \simeq V_{F}\left(\vec{r}, \widetilde{P}^{(F)}\right)+V_{e}^{\text {crystal }}(\vec{r})+V_{e}^{H}\left(\vec{r},\left\{\widetilde{P}^{(F)}\right\}\right),
$$

where $V_{F}$ describes the binary interaction of the active electron with the active fluorine site, $V^{\text {crystal }}$ is the interaction with the crystalline environment, and $V_{e}^{H}$ is the screened Coulomb interaction with holes at the passive fluorine sites $(\vec{a} \neq \overrightarrow{0})$ :

$$
V_{e}^{H}\left(\vec{r},\left\{\widetilde{P}^{(F)}\right\}\right)=-(1-\chi) \sum_{\vec{a} \neq \overrightarrow{0}} \frac{P_{a}^{(F)}}{|\vec{r}-d \vec{a}|} .
$$

The interaction potentials appearing in Eqs. (3.14) and (3.15) depend on the occupation numbers in both the projectile and the target. As this number may change in the process, we label them by the occupation number of the spectator electrons (i.e., the passive electrons), $\widetilde{P}$, rather than the total occupation. For example, in the calculation of capture, the active electron is initially localized at the $F$ site at $\vec{a}$ and $\widetilde{P}_{a}^{(F)}=P_{a}^{(F)}+1$. Conversely, for loss $\widetilde{P}_{a}^{(F)}=P_{a}^{(F)}$. Analogous definitions hold for the interaction potential of the active electron with the projectile, $V_{p e}$, and with the projectileinduced image charge, $V_{p e}^{I}$.

For the interaction potential between an electron and an isolated fluorine-ion core, we use the independent-particle model (IPM) potentials of Ref. [25]. These potentials are designed such that the eigenenergies of the single-particle Schrödinger equation of all occupied orbitals in the groundstate configuration agree well with the orbital energies of the Hartree-Fock solution of the many-electron problem. The potential has the following form:

$$
V_{F}\left(r, \widetilde{P}^{(F)}\right)=-\frac{\left(Z_{t}-\widetilde{P}^{(F)}\right) \Omega(r)}{r}-\frac{\widetilde{P}^{(F)}}{r},
$$

with the screening function

$$
\Omega(r)=\left[\eta\left(e^{r / \xi}-1\right)+1\right]^{-1} .
$$

$Z_{t}$ denotes the target nuclear charge $\left(Z_{t}=9\right.$ for fluorine), and the parameters $\xi$ and $\eta$ depend on the hole population of the ion, $\widetilde{P}^{(F)}$. For example, for $\mathrm{F}^{-}$(corresponding to first electron capture from the surface), $\xi=0.8$ and $\eta=1.888$, and for $\mathrm{F}^{0}$ (corresponding to capture of a second electron from the same F site), $\xi=0.663$ and $\eta=1.71$ [25]. 
The interaction with the other sites of the crystal, $V_{e}^{\text {crystal }}$, is decomposed into the Madelung potential of the ionic lattice, $V_{M a d}$, and the correlation contribution $V_{c o r r}$

$$
V_{e}^{\text {crystal }}(\vec{r})=V_{\text {Mad }}(\vec{r})+V_{\text {corr }}(\vec{r})
$$

The Madelung potential is the sum of the Coulomb interactions with all static crystal charges except for the one at the origin. When the active electron is at large distances from the surface, this interaction should behave as

$$
V_{M a d}(\vec{r}) \underset{r \rightarrow \infty}{\rightarrow}-\frac{1}{r}
$$

since a hole is left behind in the solid. To reach this limit we set the Madelung potential to

$$
V_{M a d}(\vec{r})=-\sum_{|\vec{a}|=1} \frac{q_{0}+\left(1-q_{0}\right) / 5}{|\vec{r}-d \vec{a}|}-\sum_{|\vec{a}|>1} \frac{q_{\vec{a}}}{|\vec{r}-d \vec{a}|} .
$$

The first sum in Eq. (3.21) runs over the five $\mathrm{Li}^{+}$ions at the nearest-neighbor sites of the active fluorine where we have increased their charge by an amount of $\left(1-q_{0}\right) / 5$, corresponding to the sharing fraction of the active electron with the neighboring $\mathrm{Li}^{+}$ions. In turn, the second sum in Eq. (3.21) runs over all other ionic sites in the solid, which have the ionic charge $q_{0}$. The particular form of $V_{M a d}$ enforces charge neutrality, and has the proper limit at large distances.

The correlation potential $V_{\text {corr }}$ accounts for the rearrangement of the charge density of the crystal as an electron is removed, and can be expressed as

$$
V_{c o r r}(\vec{r})=V_{e}^{S I}(z)+V_{s c r}(r) .
$$

The first contribution is the self-image potential of the electron with the asymptotic behavior

$$
V_{e}^{S I}(z) \underset{z \rightarrow \infty}{\rightarrow}-\frac{\chi}{4 z}
$$

The second contribution to $V_{\text {corr }}$ in Eq. (3.22) is the "screening" of the hole left in the solid, which tends asymptotically to

$$
V_{s c r}(r) \underset{r \rightarrow \infty}{\rightarrow} \frac{\chi}{r}
$$

In the opposite limit $(r \rightarrow 0)$, one can estimate the correlation potential from $V_{e}^{\text {crystal }}$ and the work function of $\mathrm{LiF}, W$ $\simeq 12 \mathrm{eV}$ [26]. The work function should correspond to the sum of the (negative) electron affinity of the "active" fluorine, $E_{a f f}=3.4 \mathrm{eV}$ [27], shifted downwards by the interaction with the remainder of the crystal, $V_{e}^{\text {crystal }}(\vec{r}=0)$. In other words,

$$
V_{e}^{\text {crystal }}(0) \simeq-W+E_{a f f} .
$$

At small distances, Eq. (3.21) yields $V_{\text {Mad }}(0)=$ - $11.3 \mathrm{eV}$. Equations (3.22) and (3.25) imply a correlation energy of

$$
E_{\text {corr }}=V_{\text {corr }}(0)=-W+E_{a f f}-V_{M a d}(0)=2.7 \mathrm{eV} .
$$

We note parenthetically that the present choice of $V_{e}^{\text {crystal }}$ differs slightly from that of Ref. [7] (our results are insensitive to this change). The present choice is motivated by a direct identification of the correlation energy. It is a wellknown fact of band-structure theory that Hartree-Fock calculations, which (due to their intrinsic mean-field character) neglect correlation, yield a valence-band edge for alkali halides, which is too low by $1-3 \mathrm{eV}[28,29]$. However, the use of the dielectric response via Eq. (3.22) allows for the inclusion of correlation effects that are due to the collective response of the valence-band electrons. The physical meaning of the positive correlation energy is that, due to the dielectric response of the surface, it takes less energy to remove an electron than estimated solely from the independent-particle potentials. Or, expressed the other way around, recombination of an electron with the hole yields less energy than expected from the independent-particle potentials because one has to "undo" the relaxation of the remaining valence-band electrons. The specific forms of the potentials $V_{e}^{S I}$ and $V_{s c r}$ used in our simulations are analogous to that of Eq. (3.12). They have the proper behavior at large distances and are smoothly extrapolated at small distances such that their sum yields the constant value $E_{\text {corr }}$ at the origin. We note that we have neglected any dependence of $V_{e}^{\text {crystal }}$ on the hole excitation.

The interaction between the electron and the projectile has been described in detail in Ref. [7]. Therefore, we just summarize the contributions introduced in Eq. (3.14). For $V_{p e}\left(\vec{r}, \vec{R},\left\{P^{(P)}\right\}\right)$, we adopt a Coulomb interaction using the effective charge introduced in Eq. (3.6),

$$
V_{p e}\left(\vec{r}, \vec{R},\left\{P^{(P)}\right\}\right)=-\frac{Q_{e f f}\left(R_{z},\left\{P^{(P)}\right\}\right)}{|\vec{r}-\vec{R}|} .
$$

In turn, the interaction of the electron with the image of the ion is chosen such that at large distances of the ion to the surface, it behaves as

$$
V_{p e}^{I}(\vec{r}, \vec{R}) \underset{R_{z} \rightarrow \infty}{\rightarrow} \frac{\chi Q_{e f f}\left(R_{z},\left\{P^{(P)}\right\}\right)}{\sqrt{\left(x-R_{x}\right)^{2}+\left(y-R_{y}\right)^{2}+\left[\operatorname{sgn}(z) z+R_{z}\right]^{2}}},
$$

where $\operatorname{sgn}(z)$ denotes the sign of $z$. At small values of $R_{z}$, we use again the functional form of Eq. (3.12), such that $V_{p e}^{I}$ approaches a constant value and the associated forces vanish.

We complete the discussion of effective potentials with the discussion of an effective "blocking" potential required within the framework of classical trajectory Monte Carlo (CTMC) simulations for the one-electron transfer rates to be presented in the following section. Note that this potential does not enter the Liouville master equation itself and would be dispensable in many-body quantum calculations of the rates $\Gamma$. Its necessity originates from the negative singulari- 


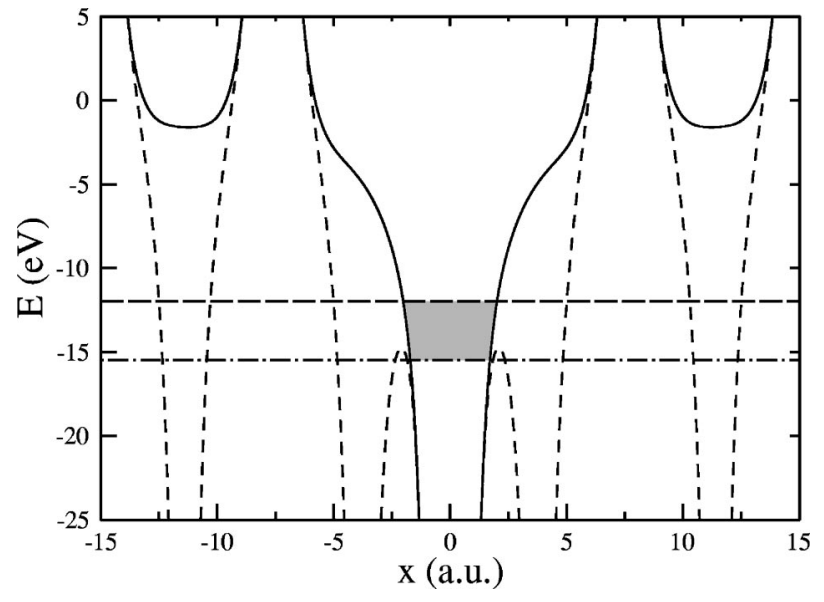

FIG. 2. Interaction potential $V_{\text {te }}$ between the active electron and a LiF surface plotted along the $(x, 0,0)$ axis: potential including blocking of the Li sites (solid line) and without blocking potential (dashed line). The horizontal dashed (dash-dotted) line represents the upper (lower) edge of the valence band. The shaded area corresponds to the allowed region of the classical valence electron in the $E-x$ plane.

ties of $V_{M a d}$ at lithium sites. In a CTMC simulation, this leads to a high probability that the electron becomes attached to the lithium ions instead of to the fluorine sites. The lack of a proper many-body quantum ground state and of the orthogonality to core states in a classical simulation leads to a spurious access of classically available phase space near the $\mathrm{Li}$ ion. In the reduction from a many-body problem to a one-electron problem, "blocking" is frequently simulated quantum mechanically using pseudopotentials for valence electrons. The pseudopotentials cut off the attractive singular potential and, for $s$-wave functions, may even display a repulsive well. In order to enforce blocking and simulate a pseudopotential for the classical electron motion, we "cut off" the Coulomb singularity of Li at $r_{c}=2.6$ a.u. and fit a function of the form $f(r)=a+b r^{6}$ such that the potential and its derivative are continuous at $r_{c}$. The value of $r_{c}$ coincides approximately with the corresponding cutoff radius for the pseudopotential for a $2 s$ electron in Li given in Ref. [30]. Figure 2 illustrates the effect of the blocking potential on the total surface potential $V_{t e}$. Without blocking of the lithium sites, an electron in the valence band (also depicted in the figure) could easily cross the potential barrier towards the neighboring Li ions. After inclusion of a blocking potential, a valence-band electron is spatially constricted to a narrow region (indicated by a gray shaded area) around the active $\mathrm{F}$ site. Note that the pseudopotential also prevents electrons with energies in the gap between valence and conduction band from entering the solid at places other than the active fluorine sites carrying a hole.

\section{ONE-ELECTRON CAPTURE AND LOSS RATES}

Capture and loss rates entering the Liouville master equation (2.8) are calculated using a CTMC method [31,32]. The CTMC method used for calculating rates should be clearly distinguished from the Monte Carlo method for integrating
Eq. (2.8). In principle, full quantum calculations for rates could be used and would be preferable which, however, in most cases are not feasible. It should also be stressed that the present CTMC method for rates in the FIA is different from the conventional CTMC method originally developed for the calculation of electron transfer and ionization cross sections in ion-atom collisions involving one active electron [31]. While in the standard CTMC method, the probability (or cross section) for a given process is determined from the classical phase-space distribution at infinite distances of the collision partners $(R \rightarrow \infty)$, or equivalently $t \rightarrow \infty$, we extract rates for a given electronic process from the differential change of the electronic phase-space distribution at a fixed position $\vec{R}$ of the projectile. Moreover, we extend our treatment to the transfer of more than one electron from a given $\mathrm{F}$ site at the surface.

The CTMC treatment of electronic transitions involving many electrons is still an open problem. One major obstacle is the fact that a classical many-electron atom spontaneously autoionizes. The root of the problem is the lack of a "quantum ground state" in a Coulomb well in classical mechanics and of the exclusion principle. Recipes to partly incorporate these quantum features in classical dynamics have been suggested $[9,33]$ and recently incorporated in a few numerical simulations for solids $[9,34]$. However, such simulations are computationally quite demanding for many electrons and are still under development. Due to these limitations, many classical simulations of a system with more than one electron have resorted to utilizing static or time-dependent screening potentials [35] (rather than describing electron-electron interactions explicitly), much like in the IPM [36]. The basic idea consists of reducing an intrinsic many-body problem to a set of many one-electron problems. In addition to selecting proper screening potentials, IPM calculations require a choice of binding energies for all active electrons involved in the simulations. The orbital binding energies of electrons in an atom are set equal to the sequential ionization potentials of the atom [32], suggesting that electrons are removed sequentially. Additional many-electron features can be approximately incorporated as discussed below.

For a LiF surface, we consider the six $2 p$ electrons per fluorine negative ion, which can be successively captured by the projectile. The binding energies of these electrons correspond to atomiclike sequential ionization potentials for atoms embedded in a crystal. While we "enforce" the classical localization of the electron through the effective interaction potentials, we take the valence-band width into account by preparing an ensemble of initial conditions of the outermost most loosely bound $2 p$ electron of $\mathrm{F}^{-}$with energies corresponding to the density of states in the LiF valence band.

\section{A. Initial configuration}

Unlike in a typical simulation for ion-atom collisions in which the electron is initially in a stationary atomic eigenstate of the target at large distances between the projectile and target nuclei, within the fixed-ion approximation [17] the initial state of the system corresponds to a "molecular" state in which the active electron is localized between the surface 


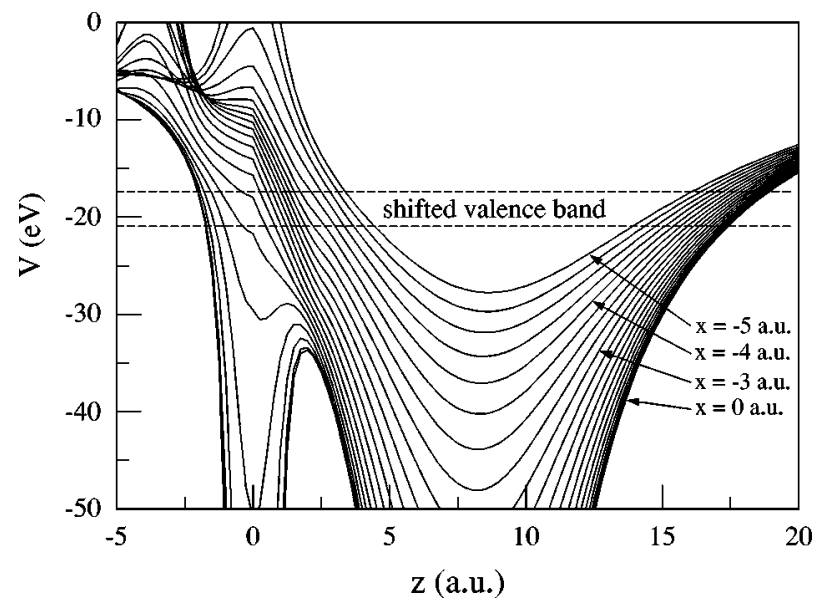

FIG. 3. Cut along the $z$ axis for $y=0$ and $x$ between -5 and 0 a.u. of the potential-energy surface $V_{e}$ for a bare projectile with $Q$ $=10$ at $\vec{R}=(0,0,8)$ a.u. The area between the two dashed lines represents the shifted valence band.

fluorine and the projectile ion. The construction of the initial electronic phase-space distribution $\rho_{i}(\vec{r}, \vec{p})$ proceeds as follows: A stationary molecular phase-space distribution is given by

$$
\rho_{\text {stationary }}(\vec{r}, \vec{p})=f\left(H-E_{i}\right)=f\left(H(\vec{r}, \vec{p})-E_{i}\right),
$$

with

$$
H=\frac{p^{2}}{2}+V_{e}(\vec{r})
$$

and where $V_{e}(\vec{r})$ denotes the total electronic potential (3.14) with all additional dependences that are constant parameters within a given CTMC run not explicitly shown, and $p$ is the momentum of the electron. The distribution function $f$ peaks at the shifted binding energy $E_{i}$ at the intermolecular distance $R$ and possesses a state-dependent width. For the most loosely bound "outer" electron, we choose for $f(E)$ the density of states of the LiF valence band [37]. In turn, for the "inner" subsequent target electrons and projectile states, we represent $f(E)$ by a narrow rectangular function with a relative width $\delta E_{i} / E_{i}=0.1$. Figure 3 presents cuts through the potential surface for a stationary state of an electron with a width corresponding to that of the valence band of LiF. If the energy of the band is higher than the potential barrier between the surface and HCI, the stationary ensemble of initial conditions is clearly molecular in character.

In order to construct now our nonstationary states for the calculation of the rates, we project this stationary state onto the initial states centered at a fluorine, $\rho_{i}^{F}$, or the projectile, $\rho_{i}^{P}$

$$
\begin{gathered}
\rho_{i}^{F}(\vec{r}, \vec{p})=C_{1} f\left(H-E_{i}^{F}\right) \mathfrak{R}(\vec{r}), \\
\rho_{i}^{P}(\vec{r}, \vec{p})=C_{2} f\left(H-E_{i}^{P}\right)[1-\mathfrak{R}(\vec{r})],
\end{gathered}
$$

where $C_{1}$ and $C_{2}$ are normalization constants and $\mathfrak{R}(\vec{r})$ is a projection function that becomes unity when the electron is ascribed to the surface and zero when it belongs to the HCI. This function can be determined from the total force $\vec{F}(\vec{r})$ $=-\vec{\nabla}_{r} V_{e}(\vec{r})$ acting on the electron. If the projection of this force onto the straight line connecting the electron with the active fluorine is larger than the projection onto the straight line connecting the electron with the projectile, $\mathfrak{R}(\vec{r})=1$, otherwise it is attributed to the projectile, i.e., $\mathfrak{R}(\vec{r})=0$.

Random initial conditions can be easily generated for any of these ensembles using the rejection method. The center of the bands of $E_{i}^{F}$ or $E_{i}^{P}$ are shifted with respect to the values for the isolated surface or the isolated projectile, $E_{i 0}^{F}$ and $E_{i 0}^{P}$,

$$
\begin{aligned}
& E_{i}^{F}=E_{i 0}^{F}+\Delta E_{0}^{F}, \\
& E_{i}^{P}=E_{i 0}^{P}+\Delta E_{0}^{P},
\end{aligned}
$$

where the $\Delta E_{0}$ 's are given in first-order perturbation theory by

$$
\begin{gathered}
\Delta E_{0}^{F}=V_{p e}(0)+V_{p e}^{I}(0), \\
\Delta E_{0}^{P}=V_{t e}(\vec{r}=\vec{R}) .
\end{gathered}
$$

The energy levels of electrons initially in the fluorine correspond to the ionization potentials $E_{I P}^{F}$ of the isolated $\mathrm{F}$ ion shifted by the interaction with the crystal,

$$
E_{i 0}^{F}=E_{I P}^{F}+V_{e}^{\text {crystal }}(0) .
$$

\section{B. Calculation of capture and loss rates}

In the CTMC method, the Hamiltonian-Liouville equation

$$
\frac{\partial \rho}{\partial t}=\{H, \rho\}
$$

governs the electronic dynamics. This Hamiltonian form of a Liouville equation for the one-electron dynamics should be distinguished from the Liouville master equation for an open system approach (2.8) coupled to the electronic many-body system. The time evolution of the initial distribution $\rho_{i}$ is calculated by Eq. (4.10) at fixed values of the ionic position $R$ and fixed occupation strings $\left\{P^{(P)}\right\}$ and $\left\{P^{(F)}\right\}$. The results are one-electron transition rates that depend parametrically on $R$, as well as the population strings $\left\{P^{(P)}\right\}$ and $\left\{P^{(F)}\right\}$. These rates are, in turn, used as input to the Liouville master equation (2.8). Equation (4.10) is solved by a Monte Carlo technique. A finite sample of $N_{t r a j}$ phase-space points distributed according to the initial density is taken, and the time evolution of each point is calculated by solving numerically the Hamilton equations of motion. The projector $\mathfrak{R}$, used to construct the target-centered and projectile-centered initial ensembles, is applied during the time propagation of the electron to determine whether the electron is still localized at the same center it originally was, or has been transferred to 


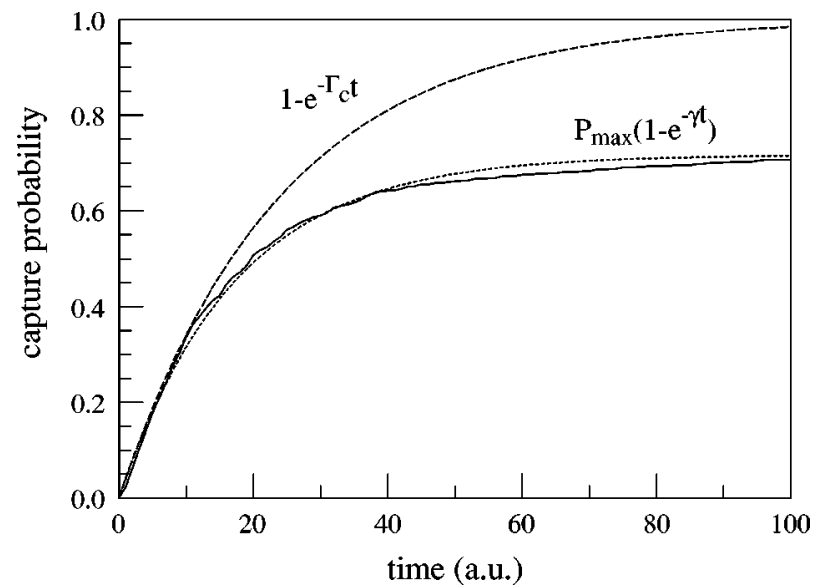

FIG. 4. Time evolution of the capture probability of the outer electron of a fluorine site at the origin for a projectile with $Q_{\text {eff }}$ $=10$ at $R_{z}=10$ a.u. incident on the $(0,0)$ zone: result of the CTMC simulation (full line), least-squares fit with the functional form $P^{C}(t)=P_{\text {max }}^{C}\left(1-e^{-\gamma t}\right)$ of Eq. (4.12) (dotted line), and function 1 $-e^{-\Gamma^{c} t}$ using the short time rate $\Gamma^{C}=\gamma P_{\max }^{C}$ (dashed line).

the other center, i.e., has crossed the barrier. The probability for electron transfer as a function of time is given by

$$
P(t)=\frac{N_{\alpha}(t)}{N_{t r a j}}
$$

where $N_{\alpha}(t)$ is the number of trajectories that have crossed the saddle within the time interval $(0, t)$. Figure 4 shows an example of $P^{\alpha}(t)$ for the case of electron capture $P^{C}$ (i.e., the electron is initially at the surface, $\alpha=C$ ). The resulting function can be easily fitted by

$$
P^{C}(t)=P_{\max }^{C}\left(1-e^{-\gamma t}\right),
$$

involving two parameters $P_{\text {max }}^{C}$ and $\gamma$. Even though the electron is energetically high above the barrier (see Fig. 3), the capture probability does not converge towards unity as $t$ $\rightarrow \infty$, but saturates at a lower value. The reason for this behavior is that the energy hypersurface of the classical electron contains regular islands (i.e., approximately conserved quantities) that remain disconnected from the saddle region within which transitions occur [38]. The parameter $\gamma$ in Eq. (4.12) is therefore not a direct measure of the electrontransfer rate. Instead, the one-electron rates are determined from the initial slope,

$$
\lim _{t \rightarrow 0} \frac{d}{d t} P^{\alpha}(t)=P^{\alpha}(t) \Gamma^{\alpha}
$$

with $\Gamma^{\alpha}=\gamma P_{\text {max }}^{\alpha}$, and $\alpha$ standing for either capture ( $\alpha$ $=C$ ) from the fluorine or loss $(\alpha=L)$ from the projectile to the fluorine site that features a hole. The approximation by the initial slope is justified by the fact that the rates are generally so high that subsequent transfers take place on a time scale before the deviation from the linear slope becomes important. The resulting rates $\Gamma^{\alpha}$ are functions in a highdimensional parameter space and depend on the strings $\{P\}$

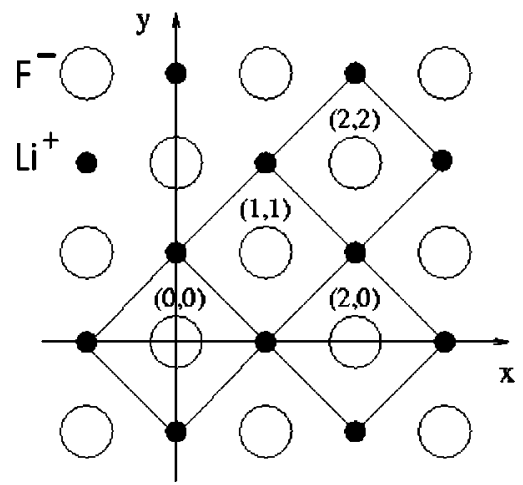

FIG. 5. Zones of the surface lattice centered around $\mathrm{F}^{-}$ions at lattice sites $\vec{a}=(i, j, 0)$. Since the third lattice coordinate is zero at the surface, we use the notation $\vec{a}=(i, j)$. The open (full) circles denote fluorine (lithium) ions.

as well as on $\vec{R}$. In order to reduce the effort of tabulation to a manageable size, we note that the one-electron transfer rates depend on the projectile populations $\left\{P^{(P)}\right\}$ only through $Q_{e f f}\left(R_{z},\left\{P^{(P)}\right\}\right)$. Therefore, the rates for capture and loss can be tabulated as a function of $Q_{e f f}$. Furthermore, we reduce the tabulation of the vectorial $\vec{R}$ dependence to a $R_{z}$ and $\vec{a}$ dependence (for $k=0$ ), where $\left(R_{x}, R_{y}\right)$ is uniformly averaged over the zones associated with the fundamental surface unit cell around each lattice site $\vec{a}$ of a fluorine ion in the surface (Fig. 5). Finally, the rates depend on the initial and final states of the electron. Since we use the fixed-ion approximation, the total energy of the electron is conserved during resonant transfer. Thus, the rates only depend on the total electronic energy. In other words, the final state of the electron is determined by its initial state (or vice versa) and both correspond to either target- or projectileshifted states [see Eqs. (4.5) and (4.6)],

$$
\begin{aligned}
& E_{f}^{F}=E_{i}^{P}, \\
& E_{f}^{P}=E_{i}^{F} .
\end{aligned}
$$

Identifying the projectile final-state occupation of the captured electron requires the mapping of the final classical atomic energy $E_{f 0}^{P}$ onto a quantum shell $n_{f}$, with orbital energy $\epsilon_{n_{f}}$ through energy binning [39]. $n_{f}$ is determined from the condition

$$
\left(\frac{n_{f}-1}{n_{f}-1 / 2}\right)^{2} \epsilon_{n_{f}-1}<E_{f 0}^{P}<\left(\frac{n_{f}}{n_{f}+1 / 2}\right)^{2} \epsilon_{n_{f}},
$$

where the orbital energy $\epsilon_{n_{f}}$ is calculated using Slater's rules [24]. That is, the orbital energy is the difference between the total energy of the $N$-electron atom (ion) projectile $E_{\text {tot }}^{P}\left(\left\{P^{(P)}\right\}\right)$ with and without one electron added to the $n_{f}$ shell:

$$
\begin{aligned}
\epsilon_{n_{f}}= & E_{\text {tot }}^{P}\left[P_{1}, P_{2}, \ldots,\left(P_{n_{f}}+1\right), \ldots\right] \\
& -E_{\text {tot }}^{P}\left[P_{1}, P_{2}, \ldots, P_{n_{f}}, \ldots\right]
\end{aligned}
$$


where

$$
E_{\text {tot }}^{P}\left(\left\{P_{n}\right\}\right)=-\sum_{n} P_{n} \frac{q_{n}\left|q_{n}\right|}{2 n^{2}}
$$

with the shell charges $q_{n}$ defined in Eq. (3.8) [for simplicity, here and in the following we drop the superscript $(P)$ for shell occupation numbers; i.e., $\left.P_{n} \equiv P_{n}^{(P)}\right]$. We use $q_{n}\left|q_{n}\right|$ rather than $q_{n}^{2}$ in order to assign shells with negative-charge positive energies (i.e., energies in the continuum). Note that in contrast to standard Slater rules for neutral atoms [24], we do not account for quantum defects. By comparison with Hartree-Fock calculations we have found that, overall, our scheme yields more realistic energies for hollow atoms. In this work we will consider only electron loss to the ground state of the solid. Therefore, $E_{i}^{P}$ is required to coincide with the shifted ground state energy of the solid (otherwise the process is blocked). Furthermore, the one-electron loss rate depends on the hole population of the active site, $P_{\vec{a}}^{(F)}$. In summary, the one-electron capture rates $\Gamma_{1}^{C}$ and loss rates $\Gamma_{1}^{L}$ are calculated as a function of $\left(Q_{e f f}, R_{z}, P_{\vec{a}}^{(F)}\right)$, where $\vec{a}$ is the lattice vector in the surface of the active fluorine site for capture or loss.

The effective capture and loss rates appearing in the Liouville master equation [Eq. (2.8)] can be, in turn, constructed from the one-electron rates, by incorporating certain manyelectron features. Two effects can be easily treated. One is the energy shift due to the many-hole interactions. That is, the resonance conditions for capture [Eq. (4.14)] can be, within the Monte Carlo solution of the Liouville master equation, modified to

$$
E_{i 0}^{F}+\Delta E_{0}^{F}+V_{e}^{H}\left(0,\left\{P^{(F)}\right\}\right)=E_{f 0}^{P}+\Delta E_{0}^{P}+V_{e}^{H}\left(\vec{R},\left\{P^{(F)}\right\}\right),
$$

where $V_{e}^{H}$ denotes the screened interaction potential between the active electron and the holes at sites different from that of the active fluorine site present at the instant of transfer. This correction leaves the tabulated one-electron rates unchanged while modifying the final-state assignment for capture. At small distances, the level shifts should saturate as the electron becomes completely shared between the ion and the surface. We estimate the saturation distance using the value of $P_{\text {max }}^{C}$ and we assume that the level shift is constant for values of $R_{z}$ such that $P_{\text {max }}^{C}>0.8$ [see Eq. (4.12)]. While the specific choice for the cutoff in $P_{\text {max }}^{C}$ is somewhat arbitrary, a large value for $P_{\max }^{C}$ is required to assure effective quasimolecular sharing.

A second feature of the many-body dynamics can be incorporated in terms of the multiplicity of the initial-state population and the number of open final-state channels. In analogy to multiplicity and blocking factors of the collision term in a quantum Boltzmann equation, we set

$$
\begin{aligned}
s \Gamma^{C}\left(\vec{R}, \Delta P_{n}\right. & \left.=1, \Delta P_{\vec{a}}^{(F)}=+1\right) \\
& =\left(6-P_{\vec{a}}^{(F)}\right)\left(1-\frac{P_{n}}{2 n^{2}}\right) \Gamma_{1}^{C}\left(Q_{e f f}, R_{z}, P_{\vec{a}}^{(F)}\right),
\end{aligned}
$$

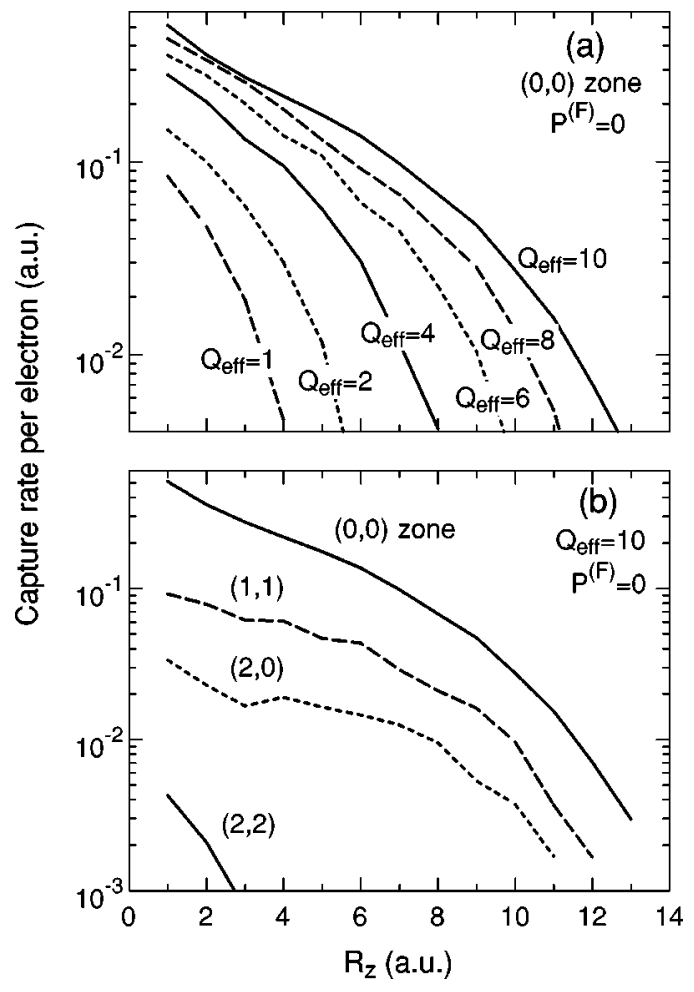

FIG. 6. Capture rate per electron from a fully occupied fluorine ion $\left(P^{(F)}=0\right)$ as a function of the ion-surface distance $R_{z}$ and assuming an empty final shell of the HCI: (a) rates for the $(0,0)$ zone and different charge states of the ion and (b) rates for a fixed charge state $\left(Q_{e f f}=10\right)$ but with the ion in different zones.

$\Gamma^{L}\left(\vec{R}, \Delta P_{n}=+1, \Delta P_{\vec{a}}^{(F)}=-1\right)=P_{n} \frac{P_{\vec{a}}^{(F)}}{6} \Gamma_{1}^{L}\left(Q_{e f f}, R_{z}, P_{\vec{a}}^{(F)}\right)$,

where $n$ is the active shell of the ion and $\vec{a}$ denotes the position of the active $F$ site. Figure 6 shows the effective capture rates per target electron, $\Gamma^{C} / 6$, into bare ions $\left(\left\{P^{(P)}=0\right\}\right)$ from a completely filled fluorine shell $\left(P_{\vec{a}}^{(F)}\right.$ $=0)$ at different active sites $\vec{a}=(i, j)$ as a function of the distance $R_{z}$ between the surface and projectile and for several values of the effective charge $Q_{\text {eff }}$. For $Q_{\text {eff }}=10$, the critical distance for the onset of capture is $R_{z} \sim 13$ a.u. For decreasing projectile charge, the critical distance and the capture rates decrease. Figure 6 also shows that the rates for capture from neighboring sites are considerably (order of magnitude) smaller than for capture from the closest fluorine ion directly "underneath" the projectile at $\vec{a}=(0,0)$. Nevertheless, capture processes from other zones $[\vec{a} \neq(0,0)]$ play an important role for neutralization of multiply charged ions at vertical incidence. The reason is that after the first capture from $\vec{a}=(0,0)$, the process competing with capture from other sites for the next step in sequential neutralization is capture from a more tightly bound electron from the same site. The latter has also a much reduced rate such that capture from more distant sites becomes competitive. Figure 7 dis- 


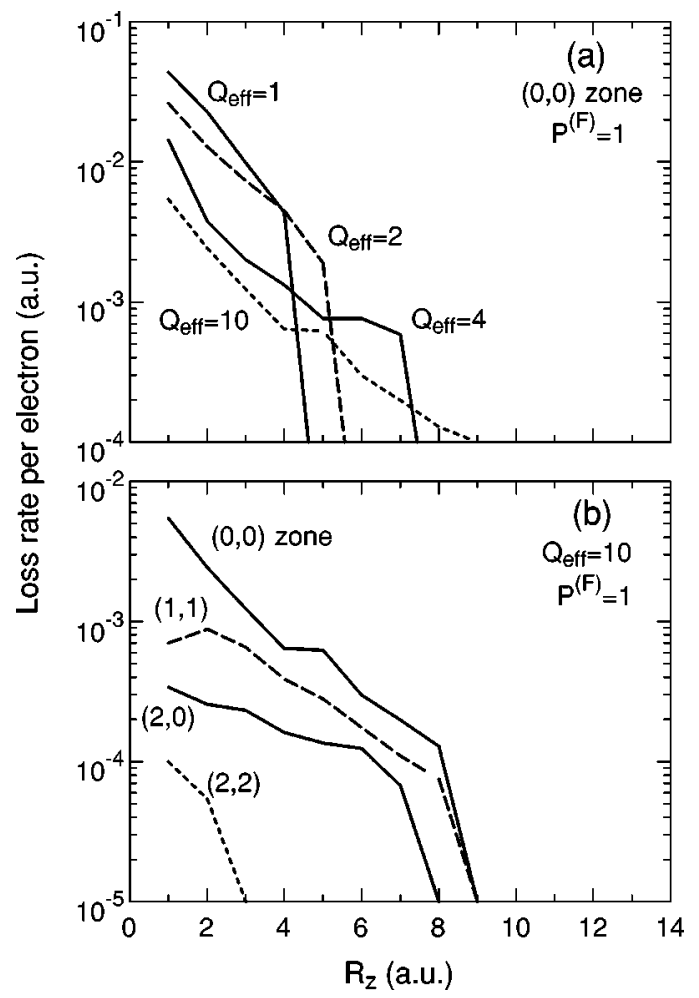

FIG. 7. Loss rate per electron $\Gamma^{L} / P_{n}$ from the projectile a fluorine ion with one vacancy $\left(P^{(F)}=1\right)$ as a function of the ion-surface distance $R_{z}$. (a) Rates for the $(0,0)$ zone and different charge states of the ion and (b) rates for a fixed charge state $\left(Q_{e f f}=10\right)$ but with the ion in different zones.

plays the loss rates per electron $\left(\Gamma^{L} / P_{n}\right)$ to different fluorine sites with one vacancy $\left(P_{a}^{(F)}=1\right)$ and for different ion charges. Overall, loss rates decrease as a function of $R_{z}$ and eventually vanish. The dependence on $R_{z}$ is, however, not always monotonic. This unusual behavior is due to the fact that the resonance condition (4.14) leads to an implicit dependence on the initial $n$ level from which the electron is lost. The critical distance for electron loss decreases with decreasing ion charge because the saddle height increases, much like that for electron capture. However, the rate at small distances increases with decreasing ion charge, because the available phase space for an electron with a given energy is smaller for lower ion charges. In general, the loss rates are small compared to the corresponding capture rates (see Fig. 6). Only for small ion charges are the loss rates of the same order of magnitude. In addition to the dependence on the effective projectile charge $Q_{\text {eff }}$ and on the zone in which the electron is localized, the rates depend also on the number of holes present. This dependence is most pronounced for holes localized at the active fluorine site (Fig. 8). The capture rates quickly decrease with increasing hole number because the remaining electrons are increasingly more tightly bound. As expected, the loss rates show the opposite behavior; it increases with increasing number of holes.

\section{Electron promotion to the continuum}

In addition to electron loss, electrons in the projectile ion can be removed by promotion to the continuum. This can
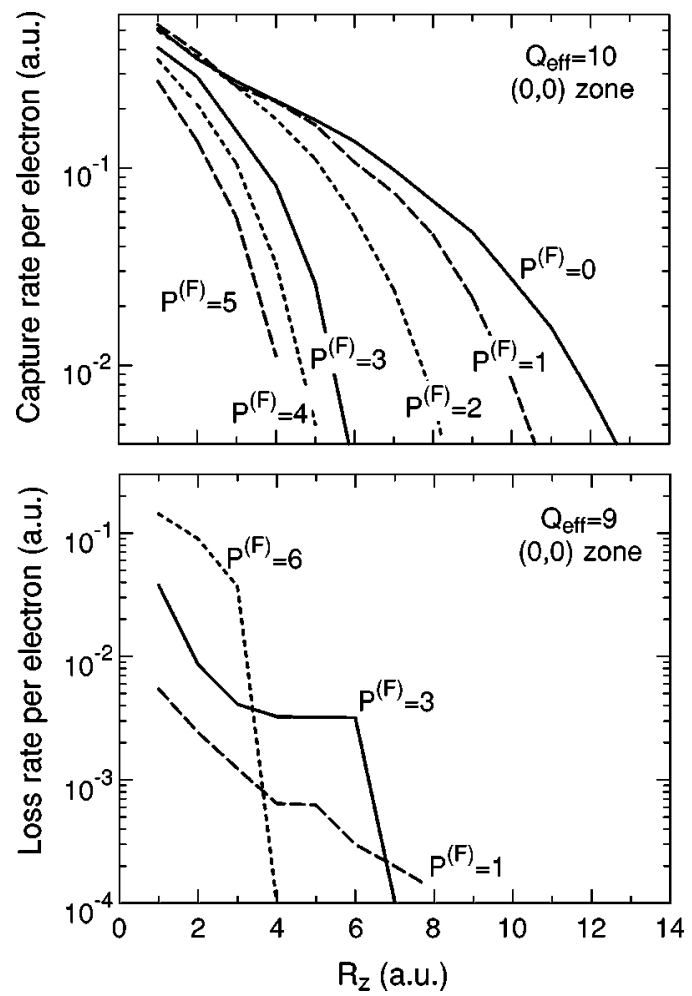

FIG. 8. Analysis of the capture (top panel) and loss rates (bottom panel) as in Figs. 6 and 7 for different hole occupation numbers of the active fluorine center site and an ion impinging on the $(0,0)$ zone.

occur for two reasons: (a) filling of inner shells and (b) single-particle level promotion. In order to account for these processes, we analyze the shifted energy of every orbital in the ion,

$$
E_{n}^{P}=\epsilon_{n}+\Delta E_{0}^{P}+V_{e}^{H}\left(\vec{R},\left\{P^{(F)}\right\}\right),
$$

after every time step. If $E_{n}^{P}$ crosses the ionization threshold $\left(E_{n}^{P}>0\right)$, one electron is removed from the $n$ shell and placed in the continuum at time $t_{I}$. The corresponding rate can be expressed as

$$
\Gamma^{I}\left(R, \Delta P_{n}=-1, \Delta P_{I}=1\right)=\delta\left(t-t_{I}\right) .
$$

By setting the threshold to $E_{n}^{P}=0$, we neglect the fact that the bottom of the unperturbed conduction band of $\mathrm{LiF}$ is about $2 \mathrm{eV}$ above the vacuum threshold. This choice can be justified by the fact that a considerable fraction of the ionized low-energy electrons remain outside the LiF surface.

\section{TWO-ELECTRON RATES}

In addition to the one-electron processes described in the preceding section, the internal state of the projectile can change due to two-electron Auger processes. We distinguish between intra-atomic and interatomic Auger processes. The former leave the internal state of the solid intact whereas the latter involve changes in both the projectile and the target. A considerable amount of quantitative information exists about 


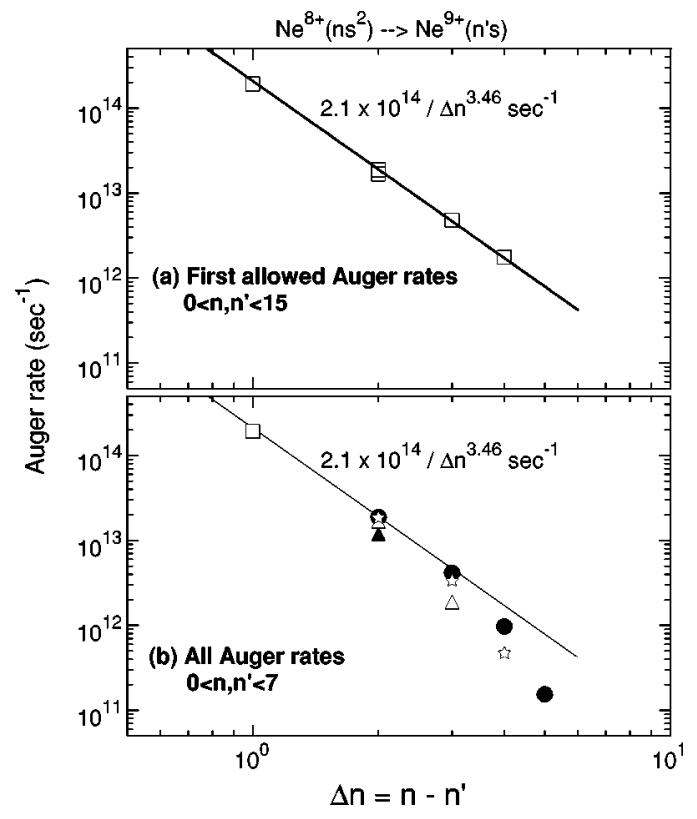

FIG. 9. Auger transition rates $\Gamma_{A I}\left(n s^{2} \rightarrow n^{\prime} s\right)$ for $\mathrm{Ne}^{8+}\left(n s^{2}\right)$ ions as a function of $\Delta n=n-n^{\prime}$. (a) First allowed transitions (open squares) and (b) all Auger transitions for $n=2$ (open squares), $n$ $=3$ (solid triangles), $n=4$ (open triangles), $n=5$ (stars), and $n$ $=7$ (solid circles) .

intraatomic Auger processes, which has been previously incorporated into many simulations of the neutralization of highly charged ions in front of surfaces. On the other hand, comparatively little information is available on interatomic Auger rates involving highly charged ions. Nonetheless, interatomic Auger processes have been found to play an important role close to the surface and for low ion charges $[40,41]$. Here we extend our previous modeling of intraatomic Auger rates in order to estimate interatomic Auger rates. The goal of the present analysis is primarily to give order-of-magnitude estimates for Auger rates valid for various ions and electronic configurations.

\section{A. Intra-atomic Auger decay}

Our estimates of intra-atomic Auger rates are an extension of previous estimates introduced in Ref. [6], which are based on calculations of Auger rates using the COWAN code $[42,43]$ for isolated ions. It was found that the first allowed and dominant Auger decay for two $s$ electrons in an upper shell $n$ decaying into an empty lower shell $n^{\prime}$ can be fitted with considerable accuracy to the functional form [6]

$$
\Gamma_{A I}\left(n s^{2} \rightarrow n^{\prime} s\right)=\frac{5.06 \times 10^{-3} \text { a.u. }}{\left(n-n^{\prime}\right)^{3.46}}=\frac{2.1 \times 10^{14} \mathrm{sec}^{-1}}{\left(n-n^{\prime}\right)^{3.46}} \text {. }
$$

The remarkable aspect of this expression is that it is valid for all $n$ and $n^{\prime}$ levels and that it is independent of the nuclear charge of the ion. Figure 9(a) illustrates the validity of Eq. (5.1) for the first energetically allowed transitions, i.e., the transition from a given $n$ to the highest $n^{\prime}$ consistent with an above-ionization-threshold energy of the second ionized

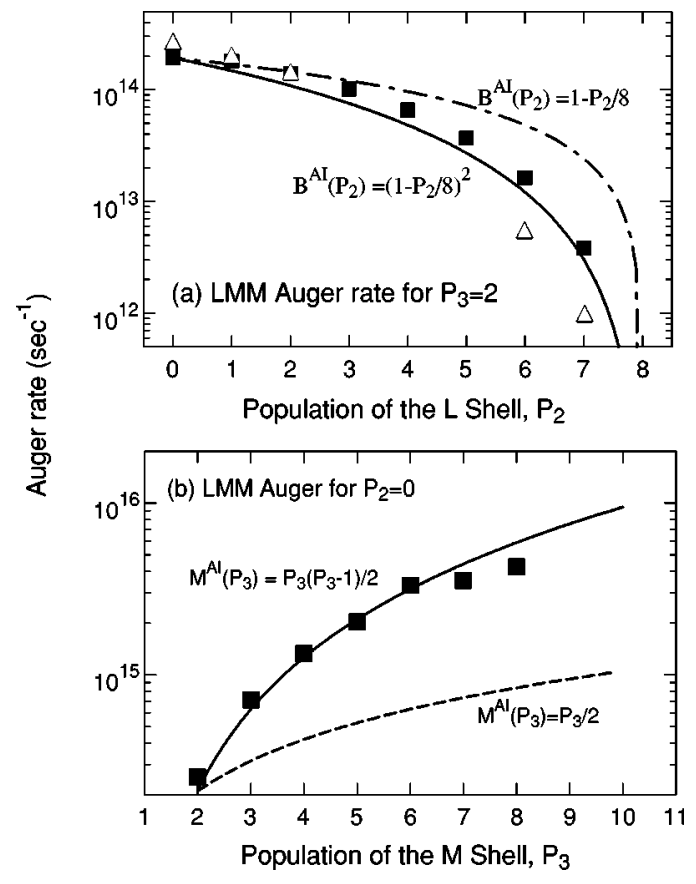

FIG. 10. (a) $L M M$ Auger transition rates as a function of the population of the $L$ shell. The solid symbols have been obtained using a $3 s^{2}$ configuration of the $M$ shell and an averaged configuration with $P_{2}$ electrons in the $L$ shell. The open symbols correspond to averaged configurations of both the upper and lower levels. (b) $L M M$ Auger rate for an empty $n=2$ shell and a configuration average of the upper shell within $s$ and $p$ orbitals. For comparison, also the multiplicity factor of Ref. [44] is shown.

electron. This transition is, in general, the fastest decay channel for the initial $n$ level. Moreover and more surprisingly, Fig. 9(b) shows that Eq. (5.1) can be used as an estimate for Auger transitions to energy levels lower than to the first allowed. In the simulations of the Liouville master equation, however, the latter do not play an important role since the relaxation of the ion proceeds predominantly as a sequence of the fastest processes.

Another important observation regarding Eq. (5.1) is that it can be used to calculate Auger rates for ions carrying more than two electrons, provided that one accounts for the multiplicity of the initial $n$ shell and the number of vacancies in the final $n^{\prime}$ shell in analogy to a quantum Boltzmann collision term. The first step in the determination of the Auger rate between many-electron levels consists of the selection whether or not the Auger transition is allowed. To this end, we first calculate the total initial and final electronic energies $E_{i}^{P}$ and $E_{f}^{P}$ using Slater's rules [Eq. (4.17)] including the shift of the projectile energy levels (4.8). The initial configuration includes $P_{n}$ and $P_{n^{\prime}}$ electrons in the $n$ and $n^{\prime}$ shells, while the final arrangement involves $P_{n}-2$ and $P_{n^{\prime}}+1$ electrons in the $n$ and $n^{\prime}$ shells. If $E_{i}^{P}-E_{f}^{P}<0$, the Auger process is energetically prohibited. Otherwise, the transition is allowed and we use as the corresponding rate

$$
\begin{gathered}
\Gamma^{A I}\left(R, \Delta P_{n}=-2, \Delta P_{n^{\prime}}=+1, \Delta P_{I}=+1\right) \\
\quad=M^{A I}\left(P_{n}\right) B^{A I}\left(P_{n^{\prime}}\right) \Gamma_{A I}\left(n s^{2} \rightarrow n^{\prime} s\right),
\end{gathered}
$$


where $M^{A I}$ and $B^{A I}$ are the multiplicity and blocking factors for initial and final states, respectively. Different factors for different systems have been previously suggested $[6,44]$. In order to determine an optimized functional form for neon, we have performed a large number of calculations for different configurations using the COWAN code [43]. Figure 10 displays the comparison of the numerically determined blocking factors with the standard Pauli blocking factor of a quantum Boltzmann collision term $\left(1-P_{n^{\prime}} / 2 n^{\prime 2}\right)$ in terms of a number of available unoccupied final states. We find that a more appropriate choice is

$$
B^{A I}\left(P_{n^{\prime}}\right)=C_{n^{\prime}}\left(1-\frac{P_{n^{\prime}}}{2 n^{\prime 2}}\right)^{2},
$$

i.e., with the square of the Pauli blocking factor, where $C_{1}$ $=0.38$ and $C_{n^{\prime}}=1$ for $n^{\prime}>1$. The factor $C_{n^{\prime}}$ has been introduced to account for the absence of the initial angularmomentum state in the final shell, i.e., $l>\left(n^{\prime}-1\right)$, which leads to a reduction of the rate (5.3). The largest correction for $\mathrm{Ne}$ ions is found for $K L L$ transitions since $2 p$ states cannot decay efficiently to a $1 s$ state. Nevertheless, using $C_{n^{\prime}} \sim 1$ becomes quite accurate for higher $n$ shells, starting already for $L M M$ transitions. The additional factor (1 $-P_{n^{\prime}} / 2 n^{\prime 2}$ ) in Eq. (5.3) whose origin is not due to Pauli blocking can be qualitatively understood in terms of the reduction of orbital overlap and of available phase space of the emitted electron when the population $P_{n^{\prime}}$ enhances the screening in the final shell $n^{\prime}$.

The initial-state multiplicity factor $M^{A I}\left(P_{n}\right)$ is well approximated by the standard binomial expression [6]

$$
M^{A I}\left(P_{n}\right)=\frac{P_{n}\left(P_{n}-1\right)}{2},
$$

where we have also shown for comparison the choice $M^{A I}$ $=P_{n} / 2$, which has been obtained from calculations of Auger rates in the bulk of metals [44]. Obviously, Eq. (5.4) appears better suited for atomic Auger transitions above the surface.

\section{B. Auger deexcitation and Auger capture}

In contrast to metal surfaces (e.g., Refs. [41,45]), little information is available on interatomic Auger rates near insulators. Thus, our estimates for interatomic Auger rates represent, perhaps, the most uncertain input into our simulation. The main goal is to analyze their relative importance in a full neutralization sequence. We therefore attempt to provide upper bounds of their actual values. Our approach employs the fact that valence electrons of the target are well localized near fluorine sites with lattice vectors $\vec{a}$. We cast the problem in terms of an interatomic Auger process in the transient quasimolecule composed of the active fluorine site and the projectile, taking into account that interatomic and intraatomic Auger processes within the same molecule should be intimately related to each other [46]. In fact, when the distance between the ion and the active site $(D=|d \vec{a}-\vec{R}|)$, is small, electrons in the molecules are "shared," and interatomic and intra-atomic Auger rates become nearly indistinguishable from each other. Molecular sharing sets in when the resonance condition [Eq. (4.14)] is met. Accordingly, for distances smaller than the sharing distance $D_{s}$, the effective Auger rates adopt the form

$$
\begin{gathered}
\Gamma^{A C}\left[D \leqslant D_{s}, \Delta P_{a}^{(F)}=-2, \Delta P_{n^{\prime}}=+1, \Delta P_{I}=+1\right]=M^{A C}\left(P_{\vec{a}}^{(F)}\right) B^{A I}\left(P_{n^{\prime}}\right) \Gamma^{A I}\left(n s^{2} \rightarrow n^{\prime} s\right), \\
\Gamma^{A D}\left[D \leqslant D_{s}, \Delta P_{a}^{(F)}=-1, \Delta P_{n}=-1, \Delta P_{n^{\prime}}=+1, \Delta P_{I}=+1\right]=M^{A D}\left(P_{a}^{(F)}\right) B^{A I}\left(P_{n^{\prime}}\right) \Gamma^{A I}\left(n s^{2} \rightarrow n^{\prime} s\right),
\end{gathered}
$$

with multiplicity factors corresponding to the number of pairs of electrons available in the initial configuration,

$$
\begin{gathered}
M^{A D}=P_{n}\left(6-P_{a}^{(F)}\right), \\
M^{A C}=\frac{1}{2}\left(6-P_{a}^{(F)}\right)\left(5-P_{a}^{(F)}\right) .
\end{gathered}
$$

Note that in $M^{A D}$ and $M^{A C}$, the number of electrons rather than the number of holes of $\mathrm{F}^{-}$enter. The initial-shell quantum number $n$ appearing in Eq. (5.5) is determined through the quasimolecular resonance condition [Eq. (4.14)]. The effective sharing distance $D_{s}$ is determined from the tabulated capture probabilities $P_{\max }^{C}$, and we set $D_{s}$ to the distance below which $P_{\max }^{C}>0.8$. To extend Eqs. (5.5) and (5.6) to distances $D>D_{s}$, we build in the large-distance behavior of the interatomic Auger rates known for metals [41] and molecules [47]. The Auger deexcitation rates should decrease as $D^{-3}$, whereas the Auger capture rate should decrease very rapidly (nearly exponentially) following the available probability density of target electrons extending out into the vacuum. We therefore extrapolate to larger distances as

$$
\begin{gathered}
\Gamma^{A C}\left[D>D_{s}, \Delta P_{a}^{(F)}=-2, \Delta P_{n^{\prime}}=+1, \Delta P_{I}=+1\right]=\rho(D) \frac{D_{s}}{D} \Gamma^{A C}\left(D=D_{s}, \ldots\right), \\
\Gamma^{A D}\left[D>D_{s}, \Delta P_{a}^{(F)}=-1, \Delta P_{n}=-1, \Delta P_{n^{\prime}}=+1 \Delta P_{I}=+1\right]=\frac{1}{2}\left[\left(\frac{D_{s}}{D}\right)^{3}+\rho(D)\right] \Gamma^{A D}\left(D=D_{s}, \ldots\right),
\end{gathered}
$$


where $\rho(D)=\exp \left[-2 \sqrt{-2 E_{i 0}^{F}}\left(D-D_{s}\right)\right]$ and $E_{i 0}^{F}$ is the orbital energy of a fluorine ion embedded in the crystal as defined in Eq. (4.9). While the smooth transitions of the rates from the molecular $\left(D<D_{s}\right)$ to the asymptotic regime ( $D$ $\gg D_{s}$ ), as implied by Eqs. (5.9) and (5.10), are certainly an oversimplification, the present functional form should provide a correct order-of-magnitude estimate.

\section{HOLE DYNAMICS}

The dynamics of the holes in the valence band created by multiple capture is of crucial importance for the neutralization dynamics. It represents one important aspect of the true many-body nature of the neutralization process. The correlation between particles and holes enters the Liouville master equation twofold. For one, the hole mobility determines the speed with which valence-band electrons are replenished after electrons have been transferred from the surface to the projectile, and therefore influence effective capture and loss rates [Eqs. (4.19) and (4.20)]. Moreover, the many-body resonance condition controlling resonant transfer molecular sharing [Eq. (4.14)] is determined by the strength of the electron-hole interaction for the distribution of holes in the surface at a given point in time.

For an estimate of the "hole-hopping time," we follow the argument given in Ref. [10] and assume a tight-binding dispersion relation for the $\mathrm{F}_{2 p}$ valence band along the [011] direction of the form

$$
E(k)=E_{0}-\beta \cos (k d \sqrt{2}),
$$

where $E_{0}$ is the energy at the center of the band. This form of the band structure is a good interpolation of the photoemission data by Himpsel et al. [37] who obtain a band halfwidth $\beta=1.75 \mathrm{eV}$. The velocity with which holes travel in the absence of strong lattice distortions is given by

$$
\begin{aligned}
v_{h}(E) & =-\frac{d E(k)}{d k}=\beta d \sqrt{2} \sin (k d \sqrt{2}) \\
& = \pm v_{h}^{h o t} \sqrt{1-\left(\frac{E-E_{0}}{\beta}\right)^{2}}
\end{aligned}
$$

A "hot" hole that is created in the center of the band has a speed of $v_{h}^{\text {hot }}=\beta d \sqrt{2} \approx 0.3$ a.u., i.e., comparable to metallic Fermi velocities. A "cold" hole produced with a spectral width of about $1 \mathrm{eV}$ near the top of the valence band has an average value of $v_{h}^{\text {cold }} \approx 0.1$ a.u. We treat the hole kinetics as an unbiased, force-free random walk. That is, the backaction of the multiply charged ion as well as of the ensembles of holes previously formed on the hole distribution by Coulomb repulsion is neglected. Therefore, our estimate for the hole speed can be considered to be a lower limit of the actual speed. Within the Monte Carlo simulation of the Liouville master equation, we follow the random walk of an ensemble of holes. The random velocity of a given stochastic hole trajectory is taken from the probability density of hole velocities,

$$
\rho_{L i F}\left(v_{h}\right)=\widetilde{C} \frac{d E}{d v_{h}} \mathcal{D}_{L i F}\left[E\left(v_{h}\right)\right]
$$

where $\widetilde{C}$ is a normalization constant and $\mathcal{D}_{L i F}(E)$ denotes the density of states of the LiF valence band [37]. From the velocity of a given hole, the nearest-neighbor site-to-site hopping rate is estimated as

$$
\Gamma_{\text {hop }}=\frac{1}{12 t_{\text {hop }}},
$$

where

$$
t_{\text {hop }}=\frac{d \sqrt{2}}{v_{h}}
$$

is the average time for hole hopping to a neighboring $\mathrm{F}$ site. The factor 1/12 in Eq. (6.4) accounts the presence of 12 nearest-neighbor F sites in the bulk. We account for the reduced number of target sites available in the random walk in the surface. The time scale of the hole hopping is in agreement with the recent coupled cluster approach of Borisov et al. [48]. Based on a self-consistent-field calculation of the hopping integral between neighboring $\mathrm{F}^{-}$ions, they have calculated the time evolution of the hole population at the surface site. Within 50 a.u., the probability that the hole is still at its origin has decreased to $30 \%$, which corresponds approximately to the hole-hopping time for a cold hole with $v_{h}^{\text {cold }} \approx 0.1$ a.u.

Since little is known about the interaction between holes, we assume that holes diffuse independently of each other. Hole-hole correlation is taken into account only in terms of blocking a given hole site for another hole with the same charge, i.e., multiple hole formation at a given site by hole hopping is suppressed. However, capture of subsequent electrons from the same fluorine site may lead to the formation of double and triple holes. Conversely, electron capture from neighboring fluorine sites can lead to the decay of a double hole into two single holes. For such events, we use the same rate as for the hole diffusion.

\section{RESULTS AND DISCUSSION}

In this section we present results of the solution of the Liouville master equation [Eq. (2.8)] with the input as described in Secs. III-VI for the interaction of $\mathrm{Ne}^{10+}$ with a $\mathrm{LiF}$ surface in either vertical or grazing incidence. In the simulation for vertical incidence, we focus on the existence (or absence) of the trampoline effect. We therefore start the ensemble of projectile trajectories at a distance of $R_{z}(t=0)$ $=20$ a.u. from the surface (which is outside the critical distance for capture), approaching the surface with a speed corresponding to a local kinetic energy of $E_{k i n}=1$ a.u. $=27 \mathrm{eV}$. This value is a lower bound for the kinetic energy due to the self-image acceleration of the $\mathrm{Ne}^{10+}$ projectile during its approach to the surface from larger distances $\left[R_{z}\right.$ $\left.\geqslant R_{z}(t=0)\right]$. Equivalently, we set the asymptotic incident kinetic energy to zero. Furthermore, to enhance the chances for observing the trampoline effect, we increase in some of 


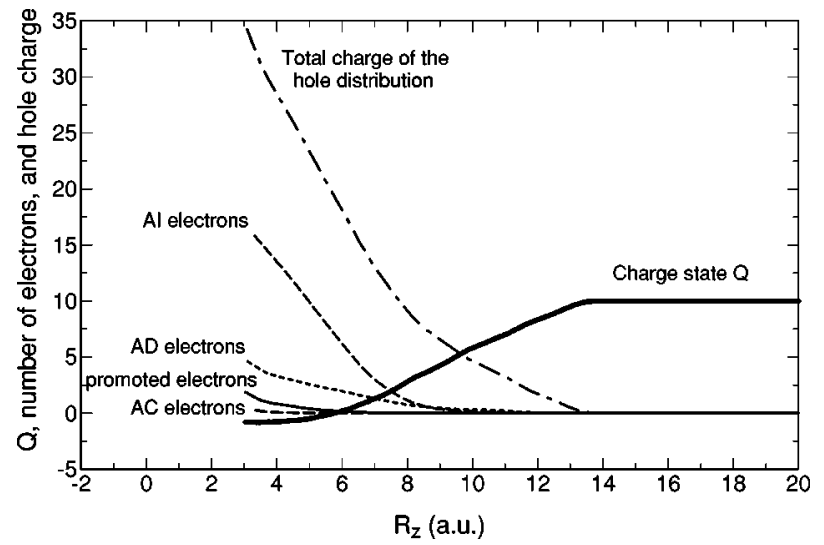

FIG. 11. Average asymptotic charge state $Q$ of an incoming $\mathrm{Ne}^{10+}$ ion impinging on a $\mathrm{LiF}$ surface in vertical incidence with $E_{k i n}=1$ a.u. as a function of the distance from the surface. Also shown are the total charge of the hole distribution, the number of promoted electrons, and the number of electrons emitted as a result of the different Auger processes [autoionization (AI), Auger deexcitation (AD), and Auger capture (AC)].

the test calculations the strength of the repulsive holeprojectile interaction [Eq. (3.5)] by neglecting any screening of the holes (i.e., we set $\chi=0$ in that equation). Figure 11 displays the charge-state evolution of the projectile as a function of the distance from the surface, $R_{z}$. Starting at the critical distance of about 14 a.u., the charge state rapidly decreases and complete neutralization (but not relaxation) is reached at about 5 a.u. The presence of the positively charged background given by the holes even allows formation of a transient negatively charged Ne ion that would not exist in the case of an isolated Ne atom. Figure 11 also demonstrates the efficiency of the different transient channels and their contributions to electron emission. The total charge of the holes created is about three times as high as the decrease in the projectile charge. This is due to the large number of electrons emitted during the autoionization by intra-atomic Auger decay. Auger deexcitation significantly contributes to neutralization and emission of electrons, while the contribution of Auger capture is barely noticeable due to the rapid exponential decrease of the rate as a function of the projectile distance. Despite the significant contribution of Auger deexcitation to the neutralization, we found that the resulting charge-state evolution is insensitive to the $\mathrm{AD}$ rate. A simulation without $\mathrm{AD}$ yields an almost identical result. The resolution of this apparent puzzle is that Auger deexcitation and the two-step process of resonant electron capture followed by intra-atomic Auger decay (AI) are competing processes. The reduction of the Auger deexcitation rates leads to an enhanced number of capture events and, in turn, via the increase in the initial-state multiplicity $M^{A I}$ [Eq. (5.4)], to a higher probability for intra-atomic Auger decay. In addition to Auger processes ( $\mathrm{AD}$ or $\mathrm{AI}$ ), electron promotion is an alternative pathway for electron emission, i.e., filling of inner shells leads to a weaker binding of outer shells that eventually become unstable. Figure 11 shows, however, that the contribution of promotion to the total electron emission is insignificant, the reason being that outer shells are rapidly

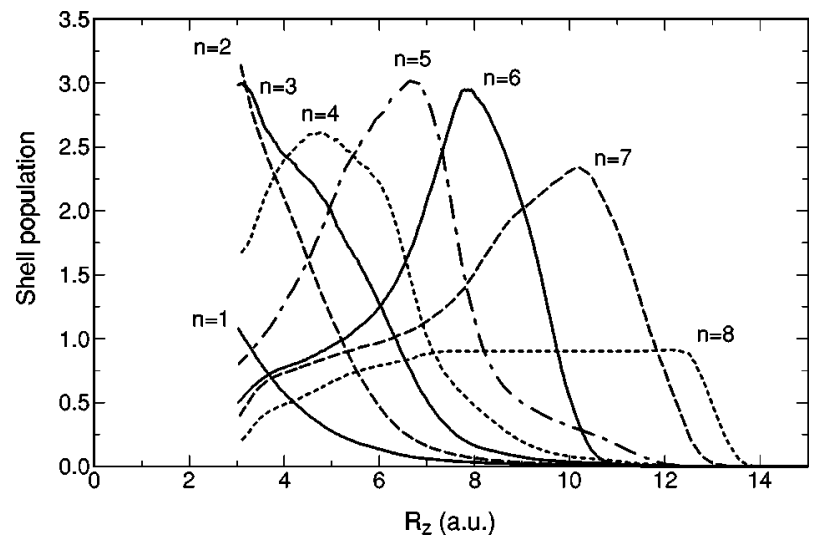

FIG. 12. Average shell occupations of an incoming $\mathrm{Ne}^{10+}$ ion impinging on a $\mathrm{LiF}$ surface in vertical incidence with $E_{\text {kin }}=1$ a.u. as a function of the distance from the surface.

depopulated by autoionization before promotion becomes efficient.

More detailed insights into the relaxation of the projectile towards its neutral ground state can be gained from the average shell occupation as a function of the projectile distance, shown in Fig. 12. The first electron is captured typically into the shell $n=8$. At slightly lower distances, electrons are captured into $n=7$. As soon as the population exceeds two electrons, Auger decay towards lower shells $(n=5)$ becomes possible while the $n=7$ shell becomes depopulated. Meanwhile, as the projectile proceeds towards smaller distances, lower $n$ shells are directly populated by resonant capture and by Auger decay from higher-lying shells. At distances smaller than 8 a.u., the shells $n=1$ and $n=2$ can be reached directly by Auger deexcitation. At a distance of 3 a.u. from the topmost layer, the $K$ and $L$ shells are, on average, half filled. The atom is still in a highly excited state with the residual electrons populating mainly the $M$ and $N$ shells. As the ion is in close proximity to the surface, resonant capture of more tightly bound electrons becomes possible. One consequence is the formation of double and triple holes, i.e., resonant capture of subsequent electrons from the same fluorine site. Figure 13 illustrates its

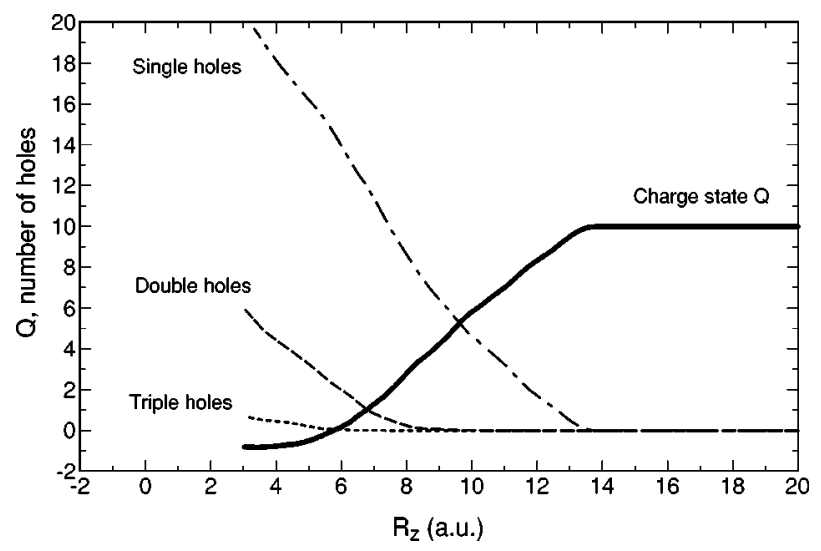

FIG. 13. Average number of single, double, and triple holes, and of the charge state of the incoming $\mathrm{Ne}^{10+}$ ion during its interaction with a $\mathrm{LiF}$ surface in vertical incidence with $E_{\text {kin }}=1$ a.u. 


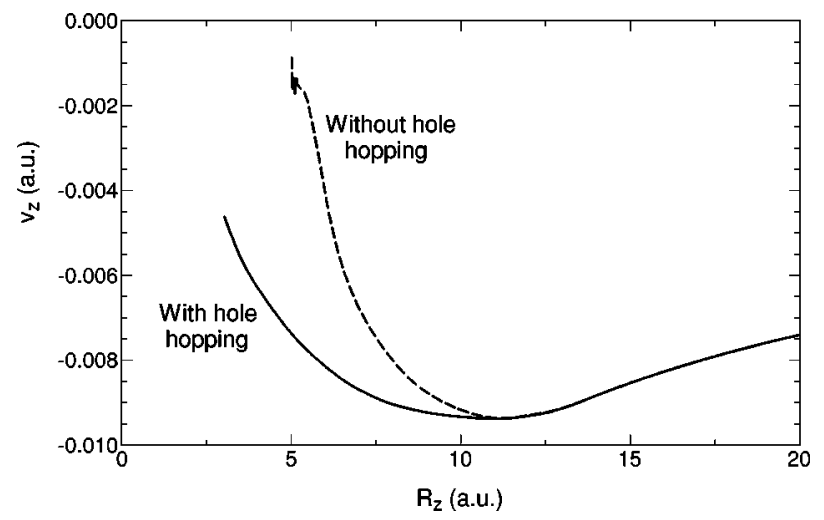

FIG. 14. Evolution of the average vertical velocity $\dot{R}_{z}$ of a $\mathrm{Ne}^{10+}$ ion with an initial energy $E_{\text {kin }}=1$ a.u. at a distance of 20 a.u. as a function of the distance from the surface. Solid line: with hole hopping as described in the text. Dashed line: hole hopping switched off. We neglect the screening of the holes in both simulations in order to enhance the hole repulsion.

contribution. If there were no hole mobility, the formation of multiple holes would be the only pathway to neutralization of the incoming projectile. However, since the holes quickly diffuse away from the site where they were created, the formation of multiple holes plays only a minor-though nonnegligible — role by allowing target electrons to be captured into deeper levels of the ion.

Focusing now on the evolution of the projectile speed, Fig. 14 displays the average velocity component perpendicular to the surface as a function of the distance. The average is always negative with $\dot{R}_{z}<0$ because it is calculated during the incoming part of the trajectory (a negative value of the velocity means movement towards the surface). At distances larger than the critical distance for first electron capture, the projectile is accelerated by the self-image interaction. As electron capture begins to contribute, the acceleration is reduced because the charge state of the projectile and its image is reduced and because of the repulsion due to holes generated by capture. At around 11 a.u., the hole repulsion starts to dominate over the image acceleration, and the projectile slows down. The repulsive force is strong enough to slow down the projectile to a velocity lower than the initial value at the distance $R_{z}(t=0)=20$ a.u., i.e., the repulsion can offset the image acceleration. However, it is, on average, not strong enough to lead to a complete stop and to a reversal of the projectile above the surface. Only $2 \%$ of all trajectories are reflected at distances larger than 3 a.u. from the topmost layer and no turning point was observed at a distance larger than 3.5 a.u. Such small distances of closest approach correspond already to the fringes of the soft binary collision regime and imply an (almost) complete neutralization of the highly charged ion. The charge-state distribution of the ion reflected from the close-collision regime is centered at low charge states $(Q \approx 1)$ and is significantly different from the notion of a trampoline-reflected ion with $Q_{\text {final }} \approx Q_{\text {initial }} / 2$. The overwhelming fraction of $98 \%$ of the projectile trajectories reach the surface and eventually penetrate it or are reflected due to hard binary collisions with surface ions. It is noteworthy that we have performed the present simulation

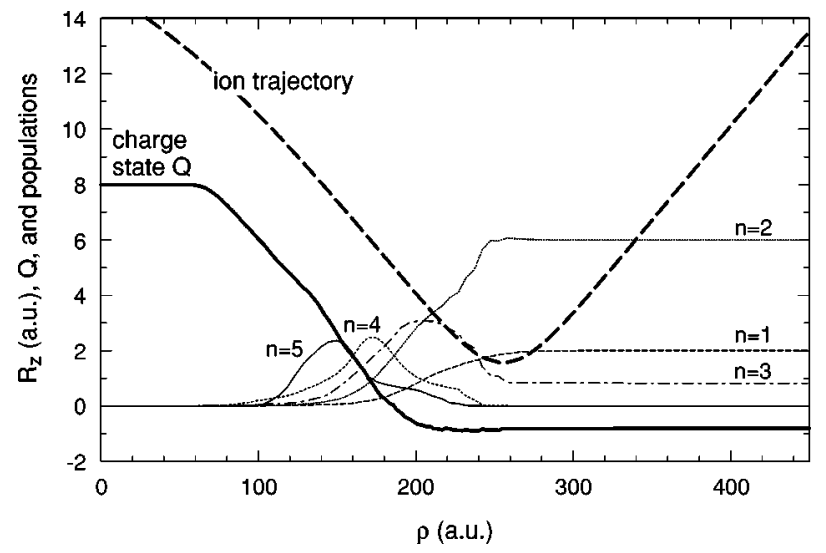

FIG. 15. Interaction of an $\mathrm{O}^{8+}$ ion approaching a $\mathrm{LiF}$ surface with $v=0.1$ a.u. in grazing incidence (angle relative to the surface $1^{\circ}$ ). Shown in the figure are the average trajectory of the ion, the average charge state of the ion, and the average shell occupations of the ion.

under the extreme assumption that the repulsive Coulomb potential of the holes in the surface is unscreened. Taking screening into account would further reduce the repulsive force considerably. In addition, we have not included the repulsion of holes by the projectile that may lead to an increased diffusion rate. Thus, we conclude that for a $\mathrm{Ne}^{10+}$ vertically incident on a $\mathrm{LiF}$ surface, the trampoline effect, i.e., the above surface reflection leaving the ion in a multiply charged state, does not exist. Our findings coincide with a recent experiment for the large angle scattering of multiply charged ions with CsI, where no signature of the trampoline effect could be detected [49]. However, Fig. 14 demonstrates that the situation could change dramatically if the hole diffusion through the crystal is suppressed. In this case, the neutralization of the projectile is incomplete and all trajectories are reflected at distances larger than 4 a.u. Therefore, the potential existence of the trampoline effect for a given material depends on the hole mobility. Only for very low or vanishing hole mobility, a partial reflection of multiply charged ions due to the trampoline effect could occur. The present simulation suggests that the hole mobility in such a case could be determined from the fraction of trampolinereflected ions.

As a second example for a solution of the Liouville master equation by Monte Carlo sampling, we present a simulation for the neutralization in grazing incidence scattering of $\mathrm{O}^{8+}$ at a LiF surface (Fig. 15). For simplicity, we employ a planar average [23] of the Moliere potential as $V_{p}^{\text {atomic }}$ in Eq. (3.4). This simplification is justified in view of the fact that the charge-state fractions have been shown to be almost independent of the azimuthal angle relative to the direction of the axial channel. We find that neutralization or relaxation is close to complete when the projectile reaches the turning point. Shell occupation and charge distribution (Fig. 15) indicate that the projectile is mostly in its neutral ground state or in its negative-ion state. This is in agreement with experiments by Meyer et al. [50] who measured high fractions of neutral and negatively charged outgoing ions and atoms for the same angle of incidence and projectile velocity. The 
ratio between neutrals and negatively charged ions is strongly dependent on parallel-velocity effects, which are neglected in the present simulation. Thus, only the sum of the fractions of neutrals and negatively charged particles can be compared with experiments. This sum exceeds 0.95 in agreement with the experiment. Our simulations explain the high probability for complete neutralization and relaxation of multiply charged ions in grazing incidence collision with a $\mathrm{LiF}$ surface, independent of the initial charge state of the ion. It is instructive to extract from Fig. 15 the absolute time scales for neutralization and relaxation. Counting the time from the first electron capture, neutralization (without relaxation) is reached for the experimental parameters from Ref. [50] at $T_{n} \simeq 30 \mathrm{fs,}$, relaxation to the ground state at $T_{n}$ $\simeq 50 \mathrm{fs}$.

It should be pointed out, however, that the good agreement with experimental data is currently restricted to low parallel velocities $v_{\|} \approx 0.1$. At higher parallel velocities, the present FIA needs to be modified. While nonadiabatic effects due to the explicit time dependence of the interaction can still be neglected for small $v_{\perp}$, the effect of the Galilei shift between target frame and projectile frame on charge exchange must be taken into account. For metals, this can be accomplished within the framework of kinematic resonances $[18-20]$ due to the Galilei-shifted Fermi sphere. For insulators, however, no comparably simple description is currently available. As a consequence, the experimentally observed decrease of the fraction of negative and neutral particles with higher projectile velocities cannot be reproduced by our present simulation. In that case, kinematic effects on the neutralization would have to be included.

In summary, we have presented a Monte Carlo simulation for stochastic trajectories representing an ensemble solution of the Liouville master equation for the many-electron dy- namics in the neutralization of highly charged ions incident on an $\mathrm{LiF}$ insulator surface. The rates for one-electron and two-electron processes entering the transport equation were estimated using CTMC calculations within the framework of the fixed-ion approximation and atomic structure codes for Auger processes. While the estimates for some of the rates obtained from data from other experiments or model calculations carry a considerable uncertainty, they represent a priori fixed input. The calculation itself does not involve any adjustable parameter. Within these limitations, our model provides for a realistic neutralization scenario. For vertical incidence of a slow $\mathrm{Ne}^{10+}$ ion, our simulations suggest that the projectile is largely neutralized (half filled $K$ and $L$ shells) when it comes within close range of the surface ( 3 a.u. of the topmost layer). The trampoline effect, i.e., the backscattering of a multiply charged ion above the surface, does not take place for this system. For grazing incidence and slow ion velocities ( $\ll 1$ a.u.), our calculations demonstrate that most reflected ions are completely neutralized-or even singly negatively charged - and in their electronic ground state, in accordance with experiments. Further extensions of our approach should address nonadiabatic effects at larger parallel velocities.

\section{ACKNOWLEDGMENTS}

We are thankful to Fred Meyer for useful discussions and for his help in the calculation of Auger rates using the code of Ref. [44]. This work was supported by the FWF (Austria), by the EU under Contract No. HPRI-CT-2001-50036, and the National Science Foundation. C.O.R. acknowledges support by the Division of Chemical Sciences, Office of Basic Energy Science, the U.S. Department of Energy, managed by UT-Battelle, LLC, under Contract No. DE-AC0500OR22725.
[1] A. Arnau, F. Aumayr, P.M. Echenique, M. Grether, W. Heiland, J. Limburg, R. Morgenstern, P. Roncin, S. Schippers, R. Schuch, N. Stolterfoht, P. Varga, T.J.M. Zouros, and H.P. Winter, Surf. Sci. Rep. 27, 113 (1997).

[2] J. Burgdörfer, in Review of Fundamental Processes and Applications of Atoms and Ions, edited by C.D. Lin (World Scientific, Singapore, 1993).

[3] S.A. Deutscher, X. Yang, and J. Burgdörfer, Phys. Rev. A 55, 466 (1997); P. Kürpick, U. Thumm, and U. Wille, ibid. 56, 543 (1997); P. Nordlander and J.C. Tully, Phys. Rev. B 42, 5564 (1990).

[4] A.G. Borisov, V. Sidis, and H. Winter, Phys. Rev. Lett. 77, 1893 (1996); A.G. Borisov and V. Sidis, Phys. Rev. B 56, 10 628 (1997).

[5] S.A. Deutscher, A.G. Borisov, and V. Sidis, Phys. Rev. A 59, 4446 (1999).

[6] J. Burgdörfer, P. Lerner, and F.W. Meyer, Phys. Rev. A 44, 5674 (1991).

[7] L. Hägg, C.O. Reinhold, and J. Burgdörfer, Phys. Rev. A 55, 2097 (1997).

[8] J. Ducrée, F. Casali, and U. Thumm, Phys. Rev. A 57, 338 (1998).
[9] J.A. Perez and R.E. Olson, in Application of Accelerators in Research and Industry, edited by J. L. Duggan and I. L. Morgan, AIP Conf. Proc. No. 475 (AIP, Woodbury, NY, 1999), p. 11.

[10] F. Aumayr, J. Burgdörfer, P. Varga, and H.P. Winter, Comments At. Mol. Phys. 34, 201 (1999).

[11] J.-P. Briand, S. Thuriez, G. Giardino, G. Borsoni, M. Froment, M. Eddrief, and C. Sébenne, Phys. Rev. Lett. 77, 1452 (1996).

[12] J.-P. Briand, L. de Billy, P. Charles, S. Essabaa, P. Briand, R. Geller, J.-P. Desclaux, S. Bliman, and C. Ristori, Phys. Rev. Lett. 65, 159 (1990).

[13] H.P. Winter and F. Aumayr, J. Phys. B 32, R39 (1999).

[14] L. Wirtz, C. Lemell, C.O. Reinhold, L. Hägg, and J. Burgdörfer, Nucl. Instrum. Methods Phys. Res. B 182, 36 (2001).

[15] H.J. Andrä, A. Simionovici, T. Lamy, A. Brenac, G. Lamboley, J.J. Bonnet, A. Fleury, M. Bonnefoy, M. Chassevent, S. Andriamonje, and A. Pesnelle, Z. Phys. D: At., Mol. Clusters 21, 135 (1991).

[16] L. Folkerts and R. Morgenstern, Europhys. Lett. 13, 377 (1990).

[17] The fixed-ion approximation goes back to the work of S. Shek- 
ter, Zh. Eksp. Teor. Fiz. 7, 750 (1937). It is justified for collision velocities where the frequency spectrum of the perturbation does not contain appreciable amplitudes for frequencies of the order of the relevant atomic frequencies [see, e.g., A. Cobas and W.E. Lamb, Jr., Phys. Rev. 65, 327 (1944)].

[18] H. Schröder, Nucl. Instrum. Methods Phys. Res. B 2, 213 (1984).

[19] J. Burgdörfer and E. Kupfer, Phys. Rev. Lett. 57, 2649 (1986).

[20] J. Los and J. Geerlings, Phys. Rep. 190, 135 (1990).

[21] J. Burgdörfer, C. Reinhold, and F. Meyer, Nucl. Instrum. Methods Phys. Res. B 98, 415 (1995).

[22] Y. Wang, P. Nordlander, and N.H. Tolk, J. Chem. Phys. 89, 4163 (1988).

[23] I. Torrens, Interatomic Potentials (Academic Press, New York, 1972).

[24] J.C. Slater, Phys. Rev. 36, 57 (1930).

[25] P.P. Szydlik and A.E.S. Green, Phys. Rev. A 9, 1885 (1974); R.H. Garvey, C.H. Jackman, and A.E.S. Green, ibid. 12, 1144 (1975).

[26] M. Piacentini and J. Anderegg, Solid State Commun. 38, 191 (1981).

[27] Handbook of Chemistry and Physics, edited by D. R. Lide (CRC Press, Boca Raton, 1995).

[28] A.B. Kunz, Phys. Rev. B 26, 2056 (1982).

[29] P. Fulde, Electron Correlations in Molecules and Solids (Springer, New York, 1995).

[30] C. Laulhé, E. Jacquet, G. Cremer, J. Pascale, Ph. Boduch, G. Rieger, M. Chantepie, and D. Lecler, Phys. Rev. A 55, 1088 (1997).

[31] R. Abrines and I.C. Percival, Proc. Phys. Soc. 88, 861 (1966).

[32] C.L. Cocke and R.E. Olson, Phys. Rep. 205, 153 (1991); D.R. Schultz, R.E. Olson, C.O. Reinhold, S. Kelbch, C. Kelbch, and H. Schmidt-Böcking, J. Phys. B 23, 3839 (1990).

[33] C.L. Kirschbaum and L. Wilets, Phys. Rev. A 21, 834 (1980); J.S. Cohen, ibid. 54, 573 (1996).

[34] K.J. LaGattuta, Opt. Express 8, 401 (2001).
[35] C.O. Reinhold and C.A. Falcon, Phys. Rev. A 33, 3859 (1986); V.J. Montemayor and G. Schiwietz, J. Phys. B 22, 2555 (1989).

[36] J.H. McGuire and L. Weaver, Phys. Rev. A 16, 41 (1977).

[37] F.J. Himpsel, L.J. Terminello, D.A. Lapiano-Smith, E.A. Eklund, and J.J. Barton, Phys. Rev. Lett. 68, 3611 (1992).

[38] N. Simonovic, J. Phys. B 30, L613 (1997).

[39] R.L. Becker and A.D. MacKellar, J. Phys. B 17, 3923 (1984).

[40] H.D. Hagstrum, Phys. Rev. 96, 336 (1954).

[41] M.A. Cazalilla, N. Lorente, R. Díez Muiño, J.-P. Gauyacq, D. Teillet-Billy, and P.M. Echenique, Phys. Rev. B 58, 13991 (1998).

[42] R.D. Cowan, The Theory of Atomic Structure and Spectra (University of California Press, Berkeley, 1981).

[43] The present rates were calculated using an extension of the COWAN code developed by S. Schippers, J. Limburg, J. Das, R. Hoekstra, and R. Morgenstern, Phys. Rev. A 50, 540 (1994).

[44] W. Huang, H. Lebius, R. Schuch, M. Grether, and N. Stolterfoht, Phys. Rev. A 56, 3777 (1997); R. Díez Muiño, N. Stolterfoht, A. Arnau, A. Salin, and P.M. Echenique, Phys. Rev. Lett. 76, 4636 (1996).

[45] S.A. Deutscher, R. Díez Muiño, A. Arnau, A. Salin, and E. Zaremba, Nucl. Instrum. Methods Phys. Res. B 182, 8 (2001).

[46] M. Corville and T.D. Thomas, Phys. Rev. A 43, 6053 (1991).

[47] A. Kay, E. Arenholz, S. Mun, F.J. Garcia de Abajo, C.S. Fadley, R. Denecke, Z. Hussain, and M.A. VanHove, Science 281, 679 (1998).

[48] A.G. Borisov, J.P. Gauyacq, V. Sidis, and A.K. Kazansky, Phys. Rev. B 63, 045407 (2001).

[49] F.W. Meyer, V.A. Morozov, J. Mrogenda, C.R. Vane, and S. Datz, in Proceedings of ICACS-19 [Nucl. Instrum Methods Phys. Res. B 193, 508 (2002)].

[50] F.W. Meyer, Q. Yan, P. Zeijlmans van Emmichoven, I.G. Hughes, and G. Spierings, Nucl. Instrum. Methods Phys. Res. B 125, 138 (1997). 